\title{
SPECTRUM AND INDEX OF TWO-SIDED ALLEN-CAHN MINIMAL HYPERSURFACES
}

\author{
FRITZ HIESMAYR
}

\begin{abstract}
The combined work of Guaraco, Hutchinson, Tonegawa and Wickramasekera has recently produced a new proof of the classical theorem that any closed Riemannian manifold of dimension $n+1 \geq 3$ contains a minimal hypersurface with a singular set of Hausdorff dimension at most $n-7$. This proof avoids the Almgren-Pitts geometric min-max procedure for the area functional that was instrumental in the original proof, and is instead based on a considerably simpler PDE minmax construction of critical points of the Allen-Cahn functional. Here we prove a spectral lower bound the hypersurfaces that arise from sequences of critical points with bounded indices. In particular, the index of two-sided minimal hypersurfaces constructed using multi-parameter Allen-Cahn min-max methods is bounded above by the number of parameters used in the construction. Finally, we point out by an elementary inductive argument how the regularity of the hypersurface follows from the corresponding result in the stable case.
\end{abstract}

\section{INTRODUCTION}

A classical theorem, due to the combined work of Almgren, Pitts and Schoen-Simon, asserts that for $n \geq 2$, every $(n+1)$-dimensional closed Riemannian manifold $M$ contains a minimal hypersurface smoothly embedded away from a closed singular set of Hausdorff dimension at most $n-7$. The original proof of this theorem is based on a highly non-trivial geometric min-max construction due to Pitts [Pit81], which extended earlier work of Almgren [Alm65]. This construction is carried out directly for the area functional on the space of hypersurfaces equipped with an appropriate weak topology, and it yields in the first instance a critical point of area satisfying a certain almost-minimizing property. This property is central to the rest of the argument, and allows to deduce regularity of the min-max hypersurface from compactness of the space of uniformly area-bounded stable minimal hypersurfaces with singular sets of dimension at most $n-7$, a result proved for $2 \leq n \leq 5$ by Schoen-Simon-Yau [SSY75] and extended to arbitrary $n \geq 2$ by Schoen-Simon [SS81]. (The Almgren-Pitts min-max construction has recently been streamlined by De Lellis and Tasnady [DT13] giving a shorter proof. However, their argument still follows Pitts' closely and is in particular based on carrying out the min-max procedure directly for the area functional on hypersurfaces.)

In recent years an alternative approach to this theorem has been developed, whose philosophy is to push the regularity theory to its limit in order

Date: July 16, 2018. 
to gain substantial simplicity on the existence part. Specifically, this approach differs from the original one in two key aspects: first, it is based on a strictly PDE-theoretic min-max construction that replaces the AlmgrenPitts geometric construction; second, for the regularity conclusions, it relies on a sharpening of the Schoen-Simon compactness theory for stable minimal hypersurfaces. The idea in this approach is to construct a minimal hypersurface as the limit-interface associated with a sequence of solutions $u=u_{i}$ to the Allen-Cahn equation

$$
\Delta u-\epsilon_{i}^{-2} W^{\prime}(u)=0
$$

on the ambient space $M$, where $W: \mathbb{R} \rightarrow \mathbb{R}$ is a fixed double-well potential with precisely two minima at \pm 1 with $W( \pm 1)=0$. Roughly speaking, if the $u_{i}$ solve (1.1) and satisfy appropriate bounds, then the level sets of $u_{i}$ converge as $\epsilon_{i} \rightarrow 0^{+}$to a stationary codimension 1 integral varifold $V$. This fact was rigorously established by Hutchinson-Tonegawa [HT00], using in part methods inspired by the earlier work of Ilmanen in the parabolic setting Ilmanen [Ilm93]. Note that $u_{i}$ solves (1.1) if and only if it is a critical point of the Allen-Cahn functional

$$
E_{\epsilon_{i}}(u)=\int_{U} \epsilon_{i} \frac{|\nabla u|^{2}}{2}+\frac{W\left(u_{i}\right)}{\epsilon_{i}} .
$$

If the solutions $u_{i}$ are additionally assumed stable with respect to $E_{\epsilon_{i}}$, then Tonegawa and Wickramasekera [TW12] proved that the resulting varifold $V$ is supported on a hypersurface smoothly embedded away from a closed singular set of Hausdorff dimension at most $n-7$, using an earlier result of Tonegawa [Ton05] which established the stability of the regular part reg $V$ with respect to the area functional. Their proof of this regularity result uses the regularity and compactness theory for stable codimension 1 integral varifolds developed by Wickramasekera [Wic14] sharpening the Schoen-Simon theory.

Stability of $u_{i}$ means that the second variation of the Allen-Cahn functional $E_{\epsilon_{i}}$ with respect to $H^{1}(M)$ is a non-negative quadratic form. More generally the index ind $u_{i}$ denotes the number of strictly negative eigenvalues of the elliptic operator $L_{i}=\Delta-\epsilon_{i}^{-2} W^{\prime \prime}\left(u_{i}\right)$, so that $u_{i}$ is stable if and only if ind $u_{i}=0$. Using min-max methods for semi-linear equations, Guaraco [Gua18] recently gave a simple and elegant construction of a solution $u_{i}$ to (1.1) with ind $u_{i} \leq 1$ and $\left\|u_{i}\right\|_{L^{\infty}} \leq 1$, and such that as $\epsilon_{i} \rightarrow 0$, the energies $E_{\epsilon_{i}}\left(u_{i}\right)$ are bounded above and below away from 0 . The lower energy bound guarantees that the resulting limit varifold $V$ is non-trivial. Since ind $u_{i} \leq 1, u_{i}$ must be stable in at least one of every pair of disjoint open subsets of $M$; similarly if ind $u_{i} \leq k$ then $u_{i}$ must be stable in at least one of every $(k+1)$-tuple of disjoint open sets. This elementary observation, originally due to Pitts in the context of minimal surfaces, together with a tangent cone analysis in low dimensions, allowed Guaraco to deduce the regularity of $V$ from the results of [TW12]. More recently still, Gaspar and Guaraco [GG18] have used $k$-parameter min-max methods to produce sequences of critical points with Morse index at most $k$, for all positive integers $k$. Our results show that this index bound is inherited by the minimal surface arising as $\epsilon_{i} \rightarrow 0$, provided it has a trivial normal bundle. 
We also point out that the regularity follows in all dimensions from the corresponding result in the stable case via an inductive argument that avoids the tangent cone analysis used in [Gua18].

Corollary. Let $M$ be a closed Riemannian manifold of dimension $n+1 \geq 3$. Let $V$ be the integral varifold arising as the limit-interface of the sequence $\left(u_{i}\right)$ of solutions to (1.1) constructed in [Gua18] (respectively in [GG18] using k-parameter min-max methods). Then $\operatorname{dim}_{\mathcal{H}} \operatorname{sing} V \leq n-7$. If $\operatorname{reg} V$ is two-sided, then its Morse index with respect to the area functional satisfies $\operatorname{ind}_{\mathcal{H}^{n}} \operatorname{reg} V \leq 1$ (respectively $\operatorname{ind}_{\mathcal{H}^{n}} \operatorname{reg} V \leq k$ ).

In min-max theory, one generally expects that the Morse index of the constructed critical point is no greater than the number of parameters used in the construction. The above corollary gives this result for the constructions of Guaraco and Gaspar-Guaraco, provided the arising hypersurface is two-sided. This was recently shown by Chodosh and Mantoulidis [CM18] to hold automatically when the ambient manifold $M$ has dimension 3 and is equipped either with a bumpy metric or has positive Ricci curvature. Building on work of Wang and Wei [WW18], Chodosh-Mantoulidis prove curvature and strong sheet separation estimates, and use these to deduce that in this three-dimensional setting the convergence of the level sets occurs with multiplicity 1 . They moreover show that in all dimensions, if the limiting surface has multiplicity 1, then its index is bounded below by the index of the $u_{\epsilon}$.

This complements our upper bound for the index, which is a direct consequence of a lower bound for $\left(\lambda_{p}\right)$, the spectrum of the elliptic operator $L_{V}=\Delta_{V}+|A|^{2}+\operatorname{Ric}_{M}(\nu, \nu)$ - the scalar Jacobi operator-in terms of $\left(\lambda_{p}^{i}\right)$, the spectra of the operators $\left(L_{i}\right)$. Establishing this spectral lower bound is our main result.

Theorem. Let $M$ be a closed Riemannian manifold of dimension $n+1 \geq 3$. Let $V$ be the integral varifold arising from a sequence $\left(u_{i}\right)$ of solutions to (1.1) with ind $u_{i} \leq k$ for some $k \in \mathbb{N}$. Then $\operatorname{dim}_{\mathcal{H}} \operatorname{sing} V \leq n-7$ and

(i) $\lambda_{p}(W) \geq \lim \sup _{i \rightarrow \infty} \lambda_{p}^{i}(W)$ for all $W \subset \subset M \backslash \operatorname{sing} V$ and $p \in \mathbb{N}$,

(ii) ind $\mathcal{H}^{n} C \leq k$ for every two-sided connected component $C \subset \operatorname{reg} V$.

Remark. The spectral lower bound of (i) also holds if the assumptions on the $u_{i}$ are weakened in a spirit similar to the work of Ambrozio, Carlotto and Sharp [ACS16], that is if instead of an index upper bound one assumes that for some $k \in \mathbb{N}$ there is $\mu \in \mathbb{R}$ such that $\lambda_{k}^{i} \geq \mu$ for all $i$. (Note that the index bound ind $u_{i} \leq k$ is equivalent to $\lambda_{k+1}^{i} \geq 0$.)

Remark. It was recently brought to our attention that a similar result had previously been proved by Le [Le11] in ambient Euclidean space, under the additional assumption that the convergence to the limit surface occurs with multiplicity 1. Adapting the methods developed in [Le11; Le15] to ambient Riemannian manifolds, Gaspar generalised our results to the case where the limit varifold is one-sided, without any assumption on multiplicity [Gas17]. Their general approach is similar to ours but subtly different, in that they instead consider the second inner variation of the Allen-Cahn functional; see also the recent work of Le and Sternberg [LS18], where similar bounds are established for other examples of eigenvalue problems. 
For the minimal hypersurfaces obtained by a direct min-max procedure for the area functional on the space of hypersurfaces (as in the AlmgrenPitts construction), index bounds have recently been established by Marques and Neves [MN16]. Both the Almgren-Pitts existence proof and the Marques-Neves proof of the index bounds are rather technically involved; in particular, the min-max construction in this setting has to be carried out in a bare-handed fashion in the absence of anything like a Hilbert space structure. In contrast, in the approach via the Allen-Cahn functional, Guaraco's existence proof is strikingly simple, and our proofs for the spectral bound and the regularity of $V$ are elementary bar the fact that they rely on the highly non-trivial sharpening of the Schoen-Simon regularity theory for stable hypersurfaces as in [Wic14].

Outline of the paper. In Section 2 we briefly expose notions from the theory of varifolds, set the context for the rest of the paper and give the statements of the main result and its corollaries. Their proof requires a number of preliminary results, which are contained in Section 3. The proof of the main result (Theorem A) is in Section 4, and is split into two parts: in the first part we prove the spectral lower bound by an inductive argument on ind $u_{i}$; this immediately implies the index upper bound. The proof of $\operatorname{dim}_{\mathcal{H}} \operatorname{sing} V \leq n-7$ is given in the second part, and uses a similar inductive argument. There are two appendices: Appendix A contains two elementary lemmas from measure theory that are used repeatedly in Section 3. Appendix B gives a proof of Proposition 3.6, which is a straight-forward adaptation of an argument used by Tonegawa for the stable case.

Acknowledgments. I would like to thank my $\mathrm{PhD}$ supervisor Neshan Wickramasekera for his encouragement and support, and Otis Chodosh for helpful conversations. This work was supported by the UK Engineering and Physical Sciences Research Council (EPSRC) grant EP/L016516/1 for the University of Cambridge Centre for Doctoral Training, the Cambridge Centre for Analysis.

\section{VARIFOLDS, STABILITY AND STATEMENT OF MAIN THEOREM}

The setting is as follows: $\left(M^{n+1}, g\right)$ is a closed (that is, compact without boundary) Riemannian manifold of dimension $n+1 \geq 3$, and $U \subset M$ is an arbitrary open subset, possibly equal to $M$ itself.

2.1. Varifolds: basic definitions. An $n$-dimensional varifold in $U$ is a Radon measure on the Grassmannian $G_{n}(U)=\{(p, E) \mid p \in U, E \subset$ $\left.T_{p} M, \operatorname{dim} E=n\right\}$ - the space of $n$-dimensional planes over points in $U$.

An important subclass are the integral varifolds, which correspond to a pair $(\Sigma, \theta)$ of a countably $n$-rectifiable set $\Sigma \subset U$ and a Borel-measurable function $\theta \in L_{\mathrm{loc}}^{1}(\Sigma, \mathbb{N})$ via

$$
V_{\Sigma, \theta}(\phi)=\int_{U} \phi\left(x, T_{x} \Sigma\right) \theta(x) \mathrm{d} \mathcal{H}^{n}(x) \quad \text { for all } \phi \in C_{c}\left(G_{n}(U)\right),
$$

where $T_{x} \Sigma$ is the $\mathcal{H}^{n}$-a.e. defined tangent space to $\Sigma$. The function $\theta$ is called the multiplicity function. 
A sequence $\left(V^{i}\right)$ converges as varifolds to $V$ if they converge weakly as Radon measures on $G_{n}(U)$, i.e. if

$$
\int_{G_{n}(U)} \phi \mathrm{d} V^{i} \rightarrow \int_{G_{n}(U)} \phi \mathrm{d} V \quad \text { for all } \phi \in C_{c}\left(G_{n}(U)\right) .
$$

The weight measure $\|V\|$ of a varifold $V$ is defined by

$$
\|V\|(\phi)=\int_{G_{n}(U)} \phi(x) \mathrm{d} V(x, S) \quad \text { for all } \phi \in C_{c}(U) .
$$

Consider an arbitrary vector field $X \in C_{c}^{1}(U, T M)$ with flow $\left(\Phi_{t}\right)$. We deform $V$ in the direction of $X$ by pushing it forward via its flow, that is

$$
\left(\Phi_{t}\right)_{*} V(\phi)=\int_{G_{n}(U)} \phi\left(\Phi_{t}(x), \mathrm{d} \Phi_{t}(x) \cdot S\right) J \Phi_{t}(x) \mathrm{d} V(x, S)
$$

for all $\phi \in C_{c}\left(G_{n}(U)\right)$, where $J \Phi_{t}(x)=\operatorname{det}\left(\mathrm{d} \Phi_{t}(x)^{*} \circ \mathrm{d} \Phi_{t}(x)\right)^{\frac{1}{2}}$ is the Jacobian of $\Phi_{t}(x)$.

Differentiating the corresponding weight measures $\left\|\left(\Phi_{t}\right)_{*} V\right\|$ yields the first variation of $V$ :

$$
\delta V(X)=\left.\frac{\mathrm{d}}{\mathrm{d} t}\right|_{t=0}\left\|\left(\Phi_{t}\right)_{*} V\right\|(U) .
$$

When $\delta V(X)=0$ for all vector fields $X \in C_{c}^{1}(U, T M)$, we say that $V$ is stationary in $U$.

By definition, the regular part of $V$ is the set of points $x \in U \cap \operatorname{spt}\|V\|$ such that in a neighbourhood of $x, \operatorname{spt}\|V\|$ is smoothly embedded in $M$. Its complement is the singular part of $V$, denoted $\operatorname{sing} V:=U \cap \operatorname{spt}\|V\| \backslash \operatorname{reg} V$. For a stationary integral varifold $V$, the Allard regularity theorem implies that $\operatorname{reg} V$ is a dense subset of $U \cap \operatorname{spt}\|V\|[\operatorname{Sim} 84$, Ch. 5].

2.2. Stability and the scalar Jacobi operator. Throughout this section $V$ will be a stationary integral $n$-varifold in $U \subset M$. We call $V$ two-sided if its regular part reg $V$ is two-sided, that is if the normal bundle $N V:=$ $N(\operatorname{reg} V)$ admits a continuous non-vanishing section. When this fails, $V$ is called one-sided. (Recall that when the ambient manifold $M$ is orientable, then $\operatorname{reg} V$ is two-sided if and only if it is orientable.)

Suppose that $V$ is two-sided, and fix a unit normal vector field $N \in$ $C^{1}(N V)$, so that every function $\phi \in C_{c}^{1}(\operatorname{reg} V)$ corresponds to a section $\phi N \in C_{c}^{1}(N V)$ and vice-versa. After extending the vector field $\phi N$ to $C_{c}^{1}(U, T M)$ - the chosen extension will not matter for our purposes - we can deform reg $V$ with respect to its flow $\left(\Phi_{t}\right)$. As $V$ is stationary, the first variation vanishes: $\delta V(\phi N)=0$. A routine calculation, the details of which can be found for instance in [Sim84, Ch. 2] shows that the second variation satisfies

$$
\begin{aligned}
\delta^{2} V(\phi N)=\left.\frac{\mathrm{d}^{2}}{\mathrm{~d} t^{2}}\right|_{t=0}\left\|\left(\Phi_{t}\right)_{*} V\right\|(U) & = \\
& \int_{U}\left|\nabla_{V} \phi\right|^{2}-\left(|A|^{2}+\operatorname{Ric}_{M}(N, N)\right) \phi^{2} \mathrm{~d}\|V\|,
\end{aligned}
$$


where $\nabla_{V}$ is the Levi-Civita connection on $\operatorname{reg} V, A$ is the second fundamental form of $\operatorname{reg} V \subset M$, and $\operatorname{Ric}_{M}$ is the Ricci curvature tensor on $M$.

The expression on the right-hand side can be defined for one-sided $V$ by replacing $N$ by an arbitrary measurable unit section $\nu: \operatorname{reg} V \rightarrow N V$, but it loses its interpretation in terms of the second variation of the area.

Definition 2.1 (Scalar second variation). The scalar second variation of a stationary integral varifold $V$ is the quadratic form $B_{V}$ defined for $\phi \in$ $C_{c}^{2}(\operatorname{reg} V)$ by

$$
B_{V}(\phi, \phi)=\int_{\operatorname{reg} V}\left|\nabla_{V} \phi\right|^{2}-\left(|A|^{2}+\operatorname{Ric}_{M}(\nu, \nu)\right) \phi^{2} \mathrm{~d}\|V\| .
$$

Remark 2.2. When $V$ is one-sided, the second variation of its area has to be measured with respect to variations in $C_{c}^{1}(N V)$-we refer to [Sim84, Ch. 2] or [CM11, Sec. 1.8] for further information on this. We called $B_{V}$ 'scalar' in order to highlight its difference with the second variation of area in this case, but emphasise that for the remainder 'second variation' refers exclusively to the quadratic form $B_{V}$ from Definition 2.7. (For the same reasons we also call the Jacobi operator $L_{V}$ 'scalar' in Definition 2.3 below, but omit this adjective in the remainder of the text.)

One can consider reg $V$ as a stationary integral varifold in its own right by identifying it with the corresponding varifold with constant multiplicity 1. Its scalar second variation

$$
B_{\operatorname{reg} V}(\phi, \phi)=\int_{\operatorname{reg} V}\left|\nabla_{V} \phi\right|^{2}-\left(|A|^{2}+\operatorname{Ric}_{M}(\nu, \nu)\right) \phi^{2} \mathrm{~d} \mathcal{H}^{n}
$$

differs from $B_{V}$ only in that the integral is with respect to the $n$-dimensional Hausdorff measure instead of $\|V\|$. This means exactly that while $B_{V}$ is 'weighted' by the multiplicity of $V$, the quadratic form $B_{\operatorname{reg} V}$ measures the variation of 'unweighted' area; we will briefly use this in Section 3.2.

After integrating by parts on $\operatorname{reg} V$, the form $B_{V}$ corresponds to the second-order elliptic operator $L_{V}=\Delta_{V}+|A|^{2}+\operatorname{Ric}_{M}(\nu, \nu)$, where $\Delta_{V}$ is the Laplacian on $\operatorname{reg} V$.

Definition 2.3 (Scalar Jacobi operator). The scalar Jacobi operator of $V$, denoted $L_{V}$, is the second-order elliptic operator

$$
L_{V} \phi=\Delta_{V} \phi+\left(|A|^{2}+\operatorname{Ric}_{M}(\nu, \nu)\right) \phi \quad \text { for all } \phi \in C^{2}(\operatorname{reg} V),
$$

where $\nu: \operatorname{reg} V \rightarrow N V$ is an arbitrary measurable unit normal vector field.

The curvature of reg $V$ can blow up as one approaches $\operatorname{sing} V$, in which case the coefficients of the operator $L_{V}$ would not be bounded. To avoid this, we restrict ourselves to a compactly contained open subset $W \subset \subset U \backslash \operatorname{sing} V$; moreover we require $W \cap \operatorname{reg} V \neq \emptyset$ to avoid vacuous statements.

We use the sign convention for the spectrum defined in [GT98, Ch. 8], where $\lambda \in \mathbb{R}$ is an eigenvalue of $L_{V}$ in $W$ if there is $\varphi \in H_{0}^{1}(W \cap \operatorname{reg} V)$ such that $L_{V} \varphi+\lambda \varphi=0$. By standard elliptic PDE theory the spectrum

$$
\lambda_{1} \leq \lambda_{2} \leq \cdots \rightarrow+\infty
$$

of $L_{V}$ in $W$ is discrete and bounded below. We will sometimes also write $\lambda_{p}(W)$ instead of $\lambda_{p}$ in order to highlight the dependence of the spectrum 
on the subset $W$. The eigenvectors of $L_{V}$ span the space $H_{0}^{1}(W \cap \operatorname{reg} V)=$ $W_{0}^{1,2}(W \cap \operatorname{reg} V)$, which we abbreviate throughout by $H_{0}^{1}$.

The index of $B_{V}$ in $W$ is the maximal dimension of a subspace of $H_{0}^{1}$ on which $B_{V}$ is negative definite; equivalently

$$
\operatorname{ind}_{W} B_{V}=\operatorname{card}\left\{p \in \mathbb{N} \mid \lambda_{p}(W)<0\right\} .
$$

Moreover ind $B_{V}:=\sup _{W}\left(\operatorname{ind}_{W} B_{V}\right)$, where the supremum is taken over all $W \subset \subset U \backslash \operatorname{sing} V$ with $W \cap \operatorname{reg} V \neq \emptyset$.

Remark 2.4. We will see in Section 3.2 that the index of $B_{V}$ coincides with the Morse index of reg $V$ with respect to the area functional, at least when $\operatorname{reg} V$ is two-sided.

2.3. Statement of main theorem. Let $\left(\epsilon_{i}\right)$ be a sequence of positive parameters with $\epsilon_{i} \rightarrow 0$ and consider an associated sequence of functions $\left(u_{i}\right)$ in $C^{3}(U)$ satisfying the following hypotheses:

(A) Every $u_{i} \in C^{3}(U)$ is a critical point of the Allen-Cahn functional

$$
E_{\epsilon_{i}}(u)=\int_{U} \epsilon_{i} \frac{|\nabla u|^{2}}{2}+\frac{W(u)}{\epsilon_{i}} \mathrm{~d} \mathcal{H}^{n+1},
$$

i.e. $u_{i}$ satisfies the equation

$$
-\epsilon_{i}^{2} \Delta u_{i}+W^{\prime}\left(u_{i}\right)=0 \text { in } U .
$$

(B) There exist constants $C, E_{0}<\infty$ such that

$$
\sup _{i}\left\|u_{i}\right\|_{L^{\infty}(U)} \leq C \text { and } \sup _{i} E_{\epsilon_{i}}\left(u_{i}\right) \leq E_{0} .
$$

(C) There exists an integer $k \geq 0$ such that the Morse index of each $u_{i}$ is at most $k$, i.e. any subspace of $C_{c}^{1}(U)$ on which the second variation

$$
\delta^{2} E_{\epsilon_{i}}\left(u_{i}\right)(\phi, \phi)=\int_{U} \epsilon_{i}|\nabla \phi|^{2}+\frac{W^{\prime \prime}\left(u_{i}\right)}{\epsilon_{i}} \phi^{2} \mathrm{~d} \mathcal{H}^{n+1}
$$

is negative definite has dimension at most $k$. We write this ind $u_{i} \leq$ $k$, and if $k=0$, say that $u_{i}$ is stable in $U$.

Remark 2.5. More generally ind $U_{U^{\prime}} u_{i}$ denotes the index of $\delta^{2} E_{\epsilon_{i}}\left(u_{i}\right)$ with respect to variations in $C_{c}^{1}\left(U^{\prime}\right)$ (or equivalently in $H_{0}^{1}\left(U^{\prime}\right)$ ) for all open subsets $U^{\prime} \subset U$. When $\operatorname{ind}_{U^{\prime}} u_{i}=0$, we say that $u_{i}$ is stable in $U^{\prime}$.

We follow Tonegawa [Ton05], using an idea originally developed by Ilmanen [Ilm93] in a parabolic setting to 'average the level sets' of $u_{i} \in C^{3}(U)$ and define a varifold $V^{i}$ by

$$
V^{i}(\phi)=\frac{1}{\sigma} \int_{U \cap\left\{\nabla u_{i} \neq 0\right\}} \epsilon_{i} \frac{\left|\nabla u_{i}(x)\right|^{2}}{2} \phi\left(x, T_{x}\left\{u_{i}=u_{i}(x)\right\}\right) \mathrm{d} \mathcal{H}^{n+1}(x)
$$

for all $\phi \in C_{c}\left(G_{n}(U)\right)$. Here $T_{x}\left\{u_{i}=u_{i}(x)\right\}$ is the tangent space to the level set $\left\{u_{i}=u_{i}(x)\right\}$ at $x \in U$, and $\sigma=\int_{-1}^{1} \sqrt{W(s) / 2} \mathrm{~d} s$ is a constant. 
Remark 2.6. In [HT00; Gua18] the varifold $V^{i}$ is defined by the slightly different expression $V^{i}(\phi)=\frac{1}{\sigma} \int_{U \cap\left\{\nabla u_{i} \neq 0\right\}}\left|\nabla w_{i}(x)\right| \phi\left(x, T_{x}\left\{u_{i}=u_{i}(x)\right\}\right) \mathrm{d} \mathcal{H}^{n+1}(x)$, with $w_{i}$ as in Theorem 2.7. The 'equipartition of energy' (2.18) from Theorem 2.7 shows that the two definitions give rise to the same limit varifold $V$ as $i \rightarrow \infty$.

The weight measures $\left\|V^{i}\right\|$ of these varifolds satisfy

$$
\left\|V^{i}\right\|(A)=\frac{1}{\sigma} \int_{A \cap\left\{\nabla u_{i} \neq 0\right\}} \epsilon_{i} \frac{\left|\nabla u_{i}\right|^{2}}{2} \mathrm{~d} \mathcal{H}^{n+1} \leq \frac{E_{0}}{2 \sigma}
$$

for all Borel subsets $A \subset U$, where the inequality follows from the energy bound in Hypothesis (B). The resulting bound $V^{i}\left(G_{n}(U)\right) \leq \frac{E_{0}}{2 \sigma}$ allows us to extract a subsequence that converges to a varifold $V$, with properties laid out in the following theorem by Hutchinson-Tonegawa [HT00].

Theorem 2.7 ([HT00]). Let $\left(u_{i}\right)$ be a sequence in $C^{3}(U)$ satisfying $H y$ potheses (A) and (B). Passing to a subsequence $V^{i} \rightarrow V$ as varifolds, and

(a) $V$ is a stationary integral varifold,

(b) $\|V\|(U)=\liminf _{i \rightarrow \infty} \frac{1}{2 \sigma} E_{\epsilon_{i}}\left(u_{i}\right)$,

(c) for all $\phi \in C_{c}(U)$ :

$$
\begin{aligned}
& \lim _{i \rightarrow \infty} \int_{U} \epsilon_{i} \frac{\left|\nabla u_{i}\right|^{2}}{2} \phi=\lim _{i \rightarrow \infty} \int_{U} \frac{W\left(u_{i}\right)}{\epsilon_{i}} \phi=\lim _{i \rightarrow \infty} \int_{U}\left|\nabla w_{i}\right| \phi, \\
& \text { where } w_{i}:=\Psi \circ u_{i} \text { and } \Psi(t):=\int_{0}^{t} \sqrt{W(s) / 2} \mathrm{~d} s .
\end{aligned}
$$

Up to a factor of $\epsilon_{i}$ the second variation $\delta^{2} E_{\epsilon_{i}}$ corresponds to the secondorder elliptic operator $L_{i}:=\Delta-\epsilon_{i}^{-2} W^{\prime \prime}\left(u_{i}\right)$. As in the discussion for the Jacobi operator, $L_{i}$ has discrete spectrum $\lambda_{1}^{i} \leq \lambda_{2}^{i} \leq \cdots \rightarrow+\infty$, which we denote by $\lambda_{p}^{i}(W)$ when we want to emphasise its dependence on the subset $W$. The following theorem is our main result.

Theorem A. Let $M^{n+1}$ be a closed Riemannian manifold, and $U \subset M$ an open subset. Let $\left(u_{i}\right)$ be a sequence in $C^{3}(U)$ satisfying Hypotheses $(A),(B)$ and $(C)$, and $V^{i} \rightarrow V$. Then $\operatorname{dim}_{\mathcal{H}} \operatorname{sing} V \leq n-7$ and

(i) $\lambda_{p}(W) \geq \lim \sup _{i \rightarrow \infty} \lambda_{p}^{i}(W)$ for all open $W \subset \subset U \backslash \operatorname{sing} V$ with $W \cap \operatorname{reg} V \neq \emptyset$ and all $p \in \mathbb{N}$,

(ii) ind $B_{V} \leq k$.

Remark 2.8. The spectral lower bound remains true if the assumptions are weakened and one assumes that for some $k \in \mathbb{N}$ there is $\mu \in \mathbb{R}$ such that

$$
\lambda_{k}^{i}(U) \geq \mu \text { for all } i \in \mathbb{N}
$$

instead of an index bound - this observation is inspired the work of AmbrozioCarlotto-Sharp [ACS16], where a similar generalisation is made in the context of minimal surfaces. One obtains the spectral bound via an inductive argument on $k$ similar to the argument in Section 4 , noting for the base case of the induction that bounds as in Corollary 3.5 hold if $\lambda_{1}^{i} \geq \mu$.

The following corollary is an immediate consequence of Theorem A.

Corollary B. If reg $V$ is two-sided, then its Morse index with respect to the area functional satisfies ind $_{\mathcal{H}^{n}} \operatorname{reg} V \leq k$. 
If $V$ is the stationary varifold arising from Guaraco's 1-parameter minmax construction [Gua18] (resp. from the $k$-parameter min-max construction of Gaspar-Guaraco [GG18]) and its regular part is two-sided, then by Corollary B its Morse index is at most 1 (resp. at most $k$ ).

\section{Preliminary Results}

The preliminary results are divided into three parts. In the first, following [Ton05] we introduce 'second fundamental forms' $A^{i}$ for the varifolds $V^{i}$ and relate them to the second variation of the Allen-Cahn functional. The last two sections are dedicated to the spectra of the operators $L_{V}=\Delta_{V}+|A|^{2}+\operatorname{Ric}_{M}(\nu, \nu)$ and $L_{i}=\Delta-\epsilon_{i}^{-2} W^{\prime \prime}\left(u_{i}\right)$.

3.1. Stability and $L^{2}$-bounds of curvature. To simplify the discussion fix for the moment a critical point $u \in C^{3}(U) \cap L^{\infty}(U)$ of the Allen-Cahn functional $E_{\epsilon}$, with associated varifold $V^{\epsilon}$ defined by (2.16).

Let $x \in U$ be a regular point of $u$, that is $\nabla u(x) \neq 0$. In a small enough neighbourhood of $x$, the level set $\{u=u(x)\}$ is embedded in $M$. Call $\Sigma \subset M$ this embedded portion of the level set, and let $A^{\Sigma}$ be its second fundamental form. We use this to define a 'second fundamental form' for $V^{\epsilon}$.

Definition 3.1. The function $A^{\epsilon}$ is defined at all $x \in U$ where $\nabla u(x) \neq 0$ by $A^{\epsilon}(x)=A^{\Sigma}(x)$.

Remark 3.2. Second fundamental forms can be generalised to the context of varifolds via the integral identity (B.21) - see Appendix B, or [Hut86] for the original account of this theory. Strictly speaking it is an abuse of language to call $A^{\epsilon}$ the 'second fundamental form' of $V^{\epsilon}$, as it satisfies this identity only up to a small error term (B.15).

By definition $\nabla_{X} Y=\nabla_{X}^{\Sigma} Y+A^{\Sigma}(X, Y)$ for all $X, Y \in C^{1}(T \Sigma)$. Making implicit use of the musical isomorphisms here and throughout the text, write $\nu^{\epsilon}(x)=\frac{\nabla u(x)}{|\nabla u(x)|}$, so that

$$
A^{\Sigma}(X, Y)=\left\langle\nabla_{X} Y, \nu^{\epsilon}\right\rangle \nu^{\epsilon}=-\left\langle Y, \nabla_{X} \nu^{\epsilon}\right\rangle \nu^{\epsilon} .
$$

Lemma 3.3. Let $x \in U$ be a regular point of $u$. Then

$$
\left|A^{\epsilon}\right|(x)^{2} \leq \frac{1}{|\nabla u|^{2}(x)}\left(\left|\nabla^{2} u\right|^{2}(x)-|\nabla| \nabla u||^{2}(x)\right),
$$

where $\nabla^{2} u(x)$ is the Hessian of $u$ at $x$.

Proof. The second fundamental form $A^{\Sigma}$ is expressed in terms of the covariant derivative $\nabla \nu^{\epsilon}$ by

$$
A^{\Sigma}=-\left.\nabla \nu^{\epsilon}\right|_{T \Sigma \otimes T \Sigma} \otimes \nu^{\epsilon} .
$$

We can express $\nabla \nu^{\epsilon}$ as

$$
\nabla \nu^{\epsilon}=\frac{\nabla^{2} u}{|\nabla u|}-\nu^{\epsilon} \otimes \frac{\nabla|\nabla u|}{|\nabla u|},
$$

whence after restriction to $T \Sigma \otimes T \Sigma$ we get

$$
A^{\Sigma}=-\left.\frac{1}{|\nabla u|} \nabla^{2} u\right|_{T \Sigma \otimes T \Sigma} \otimes \nu^{\epsilon} .
$$


On the other hand $\nabla|\nabla u|=\left\langle\nabla^{2} u, \nu^{\epsilon}\right\rangle$ where $\nabla u \neq 0$, so after decomposing the Hessian $\nabla^{2} u$ in terms of its action on $T \Sigma$ and $N \Sigma$, we obtain

$$
\left|\nabla^{2} u\right|^{2}-|\nabla| \nabla u||^{2}=|\nabla u|^{2}\left|A^{\Sigma}\right|^{2}+\left.\left|\nabla^{2} u\right|_{T \Sigma \otimes N \Sigma}\right|^{2} \geq|\nabla u|^{2}\left|A^{\epsilon}\right|^{2} .
$$

When considering the second variation, it somewhat simplifies notation to rescale the energy as $\mathcal{E}_{\epsilon}=\epsilon^{-1} E_{\epsilon}$. Its second variation is $\delta^{2} \mathcal{E}_{\epsilon}(u)(\phi, \phi)=$ $\int_{U}|\nabla \phi|^{2}+\frac{W^{\prime \prime}(u)}{\epsilon^{2}} \phi^{2}$, defined for all $\phi \in C_{c}^{1}(U)$, which by a density argument can be extended to $H_{0}^{1}(U)$. The following identity will be useful throughout; a proof can be found in either of the indicated sources.

Lemma 3.4 ([FSV13; Ton05]). Let $u \in C^{3}(U) \cap L^{\infty}(U)$ be a critical point of $E_{\epsilon}$. For all $\phi \in C_{c}^{1}(U)$,

$$
\begin{aligned}
& \delta^{2} \mathcal{E}_{\epsilon}(u)(|\nabla u| \phi,|\nabla u| \phi)= \\
& \quad \int_{U}|\nabla u|^{2}|\nabla \phi|^{2}-\left(\left|\nabla^{2} u\right|^{2}-\left.|\nabla| \nabla u\right|^{2}+\operatorname{Ric}_{M}(\nabla u, \nabla u)\right) \phi^{2} \mathrm{~d} \mathcal{H}^{n+1} .
\end{aligned}
$$

Combining (3.7) with the $\left\|V^{\epsilon}\right\|$-a.e. bound (3.2) yields for all $\phi \in C_{c}^{1}(U)$

$$
\frac{\epsilon}{2 \sigma} \delta^{2} \mathcal{E}_{\epsilon}(u)(|\nabla u| \phi,|\nabla u| \phi) \leq \int|\nabla \phi|^{2}-\left(\left|A^{\epsilon}\right|^{2}+\operatorname{Ric}_{M}\left(\nu^{\epsilon}, \nu^{\epsilon}\right)\right) \phi^{2} \mathrm{~d}\left\|V^{\epsilon}\right\| .
$$

When $u$ is stable, that is when $\delta^{2} \mathcal{E}_{\epsilon}(u)$ is non-negative, then this identity yields $L^{2}\left(V^{\epsilon}\right)$-bounds for $A^{\epsilon}$.

Corollary 3.5. There is a constant $C=C(M)>0$ such that if $u \in C^{3}(U) \cap$ $L^{\infty}(U)$ is a critical point of $E_{\epsilon}$ and is stable in an open ball $B(x, r) \subset U$ of radius $r \leq 1$ then

$$
\int_{B\left(x, \frac{r}{2}\right)}\left|A^{\epsilon}\right|^{2} \mathrm{~d}\left\|V^{\epsilon}\right\| \leq \frac{C}{r^{2}}\left\|V^{\epsilon}\right\|(B(x, r)) .
$$

Proof. The Ricci curvature term in (3.8) can be bounded by some constant $C(M) \geq 1$ as the ambient manifold $M$ is closed, so $\int_{B(x, r)}\left|A^{\epsilon}\right|^{2} \phi^{2} \mathrm{~d}\left\|V^{\epsilon}\right\| \leq$ $C(M) \int \phi^{2}+|\nabla \phi|^{2} \mathrm{~d}\left\|V^{\epsilon}\right\|$ for all $\phi \in C_{c}^{1}(B(x, r))$. Plug in a cut-off function $\eta \in C_{c}^{1}(B(x, r))$ with $\eta=1$ in $B\left(x, \frac{r}{2}\right)$ and $|\nabla \eta| \leq 3 r^{-1}$ to obtain the desired inequality.

We now turn to a sequence $\left(u_{i}\right)$ of critical points satisfying Hypotheses $(\mathrm{A})-(\mathrm{C})$. If the $u_{i}$ are stable in a ball as in Corollary 3.5 , then the uniform weight bounds (2.17) imply uniform $L^{2}\left(V^{i}\right)$-bounds of the second fundamental forms, which we denote $A^{i}$ from now on. Under these conditions the $A^{i}$ converge weakly to the second fundamental form $A$ (in the classical, smooth sense) of $\operatorname{reg} V$.

Proposition 3.6. Let $W \subset \subset U \backslash \operatorname{sing} V$ be open with $W \cap \operatorname{reg} V \neq \emptyset$. If $\sup _{i} \int_{W}\left|A^{i}\right|^{2} \mathrm{~d}\left\|V^{i}\right\|<+\infty$, then passing to a subsequence $A^{i} \mathrm{~d} V^{i} \rightarrow A \mathrm{~d} V$ weakly as Radon measures on $G_{n}(W)$, and

$$
\int_{W}|A|^{2} \mathrm{~d}\|V\| \leq \liminf _{i \rightarrow \infty} \int_{W}\left|A^{i}\right|^{2} \mathrm{~d}\left\|V^{i}\right\|,
$$

where $A$ is the second fundamental form of $\operatorname{reg} V \subset M$. 
The weak subsequential convergence follows immediately from compactness of Radon measures; the main difficulty is to show that the weak limit is $A \mathrm{~d} V$. The proof is a straight-forward adaptation of the argument used for the stable case in [Ton05]; we present a complete argument in Appendix B for the reader's convenience.

3.2. Spectrum of $L_{V}$ and weighted min-max. Throughout we restrict ourselves to a compactly contained open subset $W \subset \subset U \backslash \operatorname{sing} V$ to avoid blow-up of the coefficients of $L_{V}$ near the singular set, and assume $W \cap$ $\operatorname{reg} V \neq \emptyset$ to avoid vacuous statements. As $W \cap \operatorname{reg} V$ is compactly contained in $\operatorname{reg} V$, it can intersect only finitely many connected components $C_{1}, \ldots, C_{N}$ of $\operatorname{reg} V$. By the constancy theorem [Sim84, Thm. 41.1] the multiplicity function $\Theta$ of a stationary integral varifold $V$ is constant on every connected component of reg $V$; we write $\Theta_{1}, \ldots, \Theta_{N}$ for the respective multiplicities of $C_{1}, \ldots, C_{N}$.

By classical theory for elliptic PDE [GT98, Ch. 8], the spectrum of $L_{V}$ has the following min-max characterisation:

$$
\lambda_{p}=\inf _{\operatorname{dim} S=p} \max _{\phi \in S \backslash\{0\}} \frac{B_{\operatorname{reg} V}(\phi, \phi)}{\|\phi\|_{L^{2}}^{2}} \text { for all } p \in \mathbb{N},
$$

where the infimum is taken over linear subspaces $S$ of $H_{0}^{1}$ (recall this is our abbreviated notation for $H_{0}^{1}(W \cap \operatorname{reg} V)$ ). From this we easily obtain a minmax characterisation that is 'weighted' by the multiplicities $\Theta_{1}, \ldots, \Theta_{N}$ in the sense that

$$
\lambda_{p}=\inf _{\operatorname{dim} S=p} \max _{\phi \in S \backslash\{0\}} \frac{B_{V}(\phi, \phi)}{\|\phi\|_{L^{2}(V)}^{2}} \text { for all } p \in \mathbb{N} .
$$

To see this, observe the following: as functions $\phi \in H_{0}^{1}$ vanish near the boundary of every connected component $C \subset \operatorname{reg} V$, the function $\phi_{C}$ on $W \cap \operatorname{reg} V$ defined by

$$
\phi_{C}= \begin{cases}\phi & \text { on } C \\ 0 & \text { on } W \cap \operatorname{reg} V \backslash C\end{cases}
$$

also belongs to $H_{0}^{1}$. Moreover

$$
B_{V}\left(\phi_{C}, \phi_{C}\right)=\Theta_{C} B_{\operatorname{reg} V}\left(\phi_{C}, \phi_{C}\right) \text { and }\left\|\phi_{C}\right\|_{L^{2}(V)}^{2}=\Theta_{C}\left\|\phi_{C}\right\|_{L^{2}}^{2},
$$

where $\Theta_{C}$ denotes the multiplicity of $C$. We then define a linear isomorphism of $H_{0}^{1}$ via normalisation by the respective multiplicities of the components. This sends $\phi \mapsto \bar{\phi}:=\sum_{j=1}^{N} \Theta_{j}^{-1 / 2} \phi_{C_{j}}$; then

$$
\frac{B_{V}(\bar{\phi}, \bar{\phi})}{\|\bar{\phi}\|_{L^{2}}^{2}}=\frac{B_{\operatorname{reg} V}(\phi, \phi)}{\|\phi\|_{L^{2}(V)}^{2}} .
$$

Therefore the 'unweighted' and 'weighted' min-max characterisations (3.11) and (3.12) are in fact equivalent. In the remainder we mainly use (3.12), and abbreviate this as $\lambda_{p}=\inf _{\operatorname{dim} S=p} \max _{S \backslash\{0\}} J_{V}$, where $J_{V}$ denotes the 'weighted' Rayleigh quotient

$$
J_{V}(\phi)=\frac{B_{V}(\phi, \phi)}{\|\phi\|_{L^{2}(V)}^{2}} \quad \text { for all } \phi \in H_{0}^{1} \backslash\{0\} .
$$


The min-max characterisation implies the following lemma, which highlights the dependence of the spectrum $\lambda_{p}(W)$ on the subset $W$.

\section{Lemma 3.7.}

(a) If $W_{1} \subset W_{2} \subset \subset U \backslash \operatorname{sing} V$, then $\lambda_{p}\left(W_{1}\right) \geq \lambda_{p}\left(W_{2}\right)$ : the spectrum is monotone decreasing.

(b) If $W_{1}, W_{2} \subset \subset U \backslash \operatorname{sing} V$ have $W_{1} \cap W_{2}=\emptyset$, then $\operatorname{ind}_{W_{1}} B_{V}+$ $\operatorname{ind}_{W_{2}} B_{V}=\operatorname{ind}_{W_{1} \cup W_{2}} B_{V}$.

(c) If $W \subset \subset U \backslash \operatorname{sing} V$ and $y \in W \cap \operatorname{reg} V$, then $\lambda_{p}(W)=\lim _{R \rightarrow 0} \lambda_{p}(W \backslash$ $\bar{B}(y, R))$.

Remark 3.8. The same properties hold for the spectrum and index of $L_{i}$, and the proof is easily modified to cover this case.

Proof. (a) This is immediate from the min-max characterisations, or simply by definition of the spectrum. Similarly for (b).

(c) By monotonicity of the spectrum we have

$$
\lambda_{p}(W \backslash \bar{B}(y, R)) \geq \lambda_{p}\left(W \backslash \bar{B}\left(y, R^{\prime}\right)\right) \geq \lambda_{p}(W)
$$

for all $R>R^{\prime}>0$. The limit as $R \rightarrow 0$ therefore exists and is bounded below by $\lambda_{p}(W)$; it remains only to show that $\lim _{R \rightarrow 0} \lambda_{p}(W \backslash \bar{B}(y, R)) \leq \lambda_{p}(W)$.

By monotonicity of the spectrum it is equivalent to show that for a fixed radius $R>0, \lim _{m \rightarrow \infty} \lambda_{p}\left(W \backslash \bar{B}\left(y, 2^{-m} R\right)\right) \leq \lambda_{p}(W)$. Let $\left(\rho_{m}\right)_{m \in \mathbb{N}}$ be a sequence in $C_{c}^{1}(B(y, R) \cap \operatorname{reg} V)$ with the following properties (such a sequence exists provided $n \geq 2$, see Remark 3.9 below):

(1) $\left.\rho_{m}\right|_{B\left(y, 2^{-m} R\right) \cap \operatorname{reg} V} \equiv 0$ and $\rho_{m} \rightarrow 1 \mathcal{H}^{n}$-a.e. in $W \backslash\{y\} \cap \operatorname{reg} V$,

(2) $\left\|\nabla_{V} \rho_{m}\right\|_{L^{2}(W \cap \operatorname{reg} V)} \rightarrow 0$.

Let a small $\delta>0$ be given and choose a family $\left(\phi_{1}, \ldots, \phi_{p}\right)$ in $C_{c}^{1}(W \cap \operatorname{reg} V)$ whose $\operatorname{span}\left(\phi_{1}, \ldots, \phi_{p}\right)=: S$ has $\max _{S \backslash 0} J_{V} \leq \lambda_{p}(W)+\delta$. Write $\rho_{m} S$ for $\operatorname{span}\left(\rho_{m} \phi_{1}, \ldots, \rho_{m} \phi_{p}\right) \subset C_{c}^{1}\left(W \backslash \bar{B}\left(y, 2^{-m} R\right) \cap \operatorname{reg} V\right)$ - for $m$ large enough the functions $\rho_{m} \phi_{i}$ are indeed linearly independent. By the weighted min$\max$ formula (3.12),

$$
\max _{\rho_{m} S \backslash 0} J_{V} \geq \lambda_{p}\left(W \backslash \bar{B}\left(y, 2^{-m} R\right)\right) .
$$

Let $t_{m} \in \mathbb{S}^{p-1} \subset \mathbb{R}^{p}$ denote the coefficients of the linear combination $t_{m} \cdot \rho_{m} \phi:=\rho_{m} \sum_{j=1}^{p} t_{m j} \phi_{j} \in \rho_{m} S$ that realises $\max _{\rho_{m} S \backslash 0} J_{V}$. Passing to a convergent subsequence $t_{m} \rightarrow t \in \mathbb{S}^{p-1} \subset \mathbb{R}^{p}$ we get $J_{V}\left(t_{m} \cdot \rho_{m} \phi\right) \rightarrow J_{V}(t \cdot \phi)$, and hence

$$
\lim _{m \rightarrow \infty} J_{V}\left(t_{m} \cdot \rho_{m} \phi\right) \leq \max _{S \backslash 0} J_{V} .
$$

On the one hand $\max _{S \backslash 0} J_{V} \leq \lambda_{p}(W)+\delta$ by our choice of $S$, on the other hand $\lim _{m \rightarrow \infty} \lambda_{p}\left(W \backslash \bar{B}\left(y, 2^{-m} R\right)\right) \leq \lim _{m \rightarrow \infty} J_{V}\left(t_{m} \cdot \rho_{m} \phi\right)$ by our choice of $t_{m}$. The conclusion follows after combining these two observations and letting $\delta \rightarrow 0$.

Remark 3.9. A sequence of functions $\left(\rho_{m}\right)$ with properties (1) and (2) exists provided $n \geq 2$, as we assume throughout. When $n \geq 3$ one can use the standard cutoff functions; for $n=2$ a more precise construction is necessary, described for instance in [EG15, Sec. 4.7]. 
3.3. Spectrum of $L_{i}$ and conditional proof of Theorem A. The main result in this section is Lemma 3.12; essentially it says that

$$
\lambda_{p}(W) \geq \limsup _{i \rightarrow \infty} \lambda_{p}^{i}(W)
$$

holds under the condition that $\sup _{i} \int_{W}\left|A^{i}\right|^{2} \mathrm{~d}\left\|V^{i}\right\|<+\infty$. What precedes it in this section are technical results required for its proof.

Again, by classical elliptic PDE theory the eigenvalues $\lambda_{p}^{i}(W)$ of $L_{i}=$ $\Delta-\epsilon_{i}^{-2} W^{\prime \prime}\left(u_{i}\right)$ on $H_{0}^{1}(W)$ have the following min-max characterisation in terms of the rescaled Allen-Cahn functional $\mathcal{E}_{\epsilon_{i}}=\epsilon_{i}^{-1} E_{\epsilon_{i}}$ :

$$
\lambda_{p}^{i}(W)=\inf _{\operatorname{dim} S=p} \max _{\phi \in S \backslash\{0\}} \frac{\delta^{2} \mathcal{E}_{\epsilon_{i}}\left(u_{i}\right)(\phi, \phi)}{\|\phi\|_{L^{2}}^{2}} \quad \text { for all } p \in \mathbb{N},
$$

where the infimum is over $p$-dimensional linear subspaces $S \subset H_{0}^{1}(W)$. Define the Rayleigh quotient $J_{i}$ by

$$
J_{i}(\phi)=\frac{\delta^{2} \mathcal{E}_{\epsilon_{i}}\left(u_{i}\right)(\phi, \phi)}{\|\phi\|_{L^{2}}^{2}} \text { for all } \phi \in H_{0}^{1}(W) \backslash\{0\},
$$

so that we can write the min-max characterisation more succinctly as $\lambda_{p}^{i}=$ $\inf _{\operatorname{dim} S=p} \max _{S \backslash\{0\}} J_{i}$.

To compare the spectrum of $L_{V}$ in $H_{0}^{1}$ with those of the operators $L_{i}$ in $H_{0}^{1}(W)$, extend functions in $C_{c}^{1}(W \cap \operatorname{reg} V)$ to $C_{c}^{1}(W)$ in the standard way, which we now describe to fix notations. Pick a small enough $0<$ $\tau<\operatorname{inj}(M)$ so that $B_{\tau} V:=\exp N_{\tau} V$ is a tubular neighbourhood of $W \cap$ $\operatorname{reg} V$, where $N_{\tau} V:=\left\{s_{p} \in N V|p \in W \cap \operatorname{reg} V,| s_{p} \mid<\tau\right\}$. We abuse notation slightly to denote points in $B_{\tau} V$ by $s_{p}$, and identify the fibre $N_{p} V$ with $\left(\exp _{p}\right)_{*} N_{p} V \subset T_{s_{p}}\left(B_{\tau} V\right)$. The distance function $d_{V}: x \in B_{\tau} V \mapsto$ $\operatorname{dist}(x, \operatorname{reg} V)$ is Lipschitz and smooth on $B_{\tau} V \backslash \operatorname{reg} V$. By the Gauss lemma $\operatorname{grad} d_{V}\left(s_{p}\right)=-s_{p} /\left|s_{p}\right|$ for all $s_{p} \in B_{\tau} V \backslash \operatorname{reg} V$. A function $\phi \in C^{1}\left(B_{\tau} V\right)$ is constant along geodesics normal to reg $V$ if $\phi\left(s_{p}\right)=\phi\left(0_{p}\right)$ for all $s_{p} \in B_{\tau} V$, or equivalently if $\left\langle\nabla \phi, \nabla d_{V}\right\rangle \equiv 0$ in $B_{\tau} V \backslash \operatorname{reg} V$.

Lemma 3.10. Any $\phi \in C_{c}^{1}(W \cap \operatorname{reg} V)$ can be extended to $C_{c}^{1}(W)$ with $\left\langle\nabla \phi, \nabla d_{V}\right\rangle \equiv 0$ in $B_{\frac{\tau}{2}} V \backslash \operatorname{reg} V$ for some $\tau=\tau(\phi)>0$.

Proof. Extend $\phi \in C_{c}^{1}(W \cap \operatorname{reg} V)$ to $B_{\tau} V$ by setting $\tilde{\phi}\left(s_{p}\right)=\phi(p)$, so that $\left\langle\nabla d_{V}, \nabla \tilde{\phi}\right\rangle \equiv 0$ in $B_{\tau} V \backslash \operatorname{reg} V$. Let $\eta \in C^{1}[0, \infty)$ be a cutoff function with $0 \leq \eta \leq 1, \eta \equiv 1$ on $[0,1 / 2)$ and $\operatorname{spt} \eta \subset[0,1)$. Then

$$
\left(\eta \circ d_{V} / \tau\right) \tilde{\phi} \in C_{c}^{1}\left(B_{\tau} V\right) \text { and }\left(\eta \circ d_{V} / \tau\right) \tilde{\phi}=\tilde{\phi} \text { on } B_{\tau / 2} V \text {. }
$$

Moreover even though $B_{\tau} V \not \subset W$ in general, as $\operatorname{spt} \phi$ is compactly contained in $W \cap \operatorname{reg} V$ we still have $\left(\eta \circ d_{V} / \tau\right) \tilde{\phi} \in C_{c}^{1}(W)$ provided $0<\tau<$ $\operatorname{dist}(\operatorname{spt} \phi, \partial W)$.

The following lemma gives an asymptotic lower bound for the Rayleigh quotient $J_{V}$ in terms of the $J_{i}$.

Lemma 3.11. Let $B_{\tau} V$ be a tubular neighbourhood of $W \cap \operatorname{reg} V$ with width $\tau>0$, and let $\left(\phi_{i}\right)_{i \in \mathbb{N}}$ be a sequence of functions in $C_{c}^{1}(W)$ with

(a) $\left\langle\nabla \phi_{i}, \nabla d\right\rangle \equiv 0$ in $W \cap B_{\tau / 2} V$ for all $i$,

(b) $\phi_{i} \rightarrow \phi$ in $C_{c}^{1}(W)$ as $i \rightarrow \infty$, where $\phi \neq 0$ in $W \cap \operatorname{reg} V$. 
If $\sup _{i} \int_{W}\left|A^{i}\right|^{2} \mathrm{~d}\left\|V^{i}\right\|<+\infty$, then $J_{V}(\phi) \geq \lim \sup _{i \rightarrow \infty} J_{i}\left(\left|\nabla u_{i}\right| \phi_{i}\right)$.

Proof. Before we start the proof proper, note that for all $\phi \in H_{0}^{1}(U)$, dividing both sides of (3.8) by $\frac{\epsilon_{i}}{2 \sigma} \int \phi^{2}\left|\nabla u_{i}\right|^{2} \mathrm{~d} \mathcal{H}^{n+1}=\int \phi^{2} \mathrm{~d}\left\|V^{i}\right\|$ yields

$$
\|\phi\|_{L^{2}\left(V^{i}\right)}^{-2} \int|\nabla \phi|^{2}-\left(\left|A^{i}\right|^{2}+\operatorname{Ric}_{M}\left(\nu_{i}, \nu_{i}\right)\right) \phi^{2} \mathrm{~d}\left\|V^{i}\right\| \geq J_{i}\left(\left|\nabla u_{i}\right| \phi\right),
$$

provided of course that $\|\phi\|_{L^{2}\left(V^{i}\right)}^{2} \neq 0$.

We treat the terms on the left-hand side separately in the calculations (1)(4) below. Once these are completed, we combine (1) with our assumption that $\|\phi\|_{L^{2}(V)}^{2} \neq 0$ to obtain that $\left\|\phi_{i}\right\|_{L^{2}\left(V^{i}\right)} \neq 0$ for large enough $i$. The conclusion follows by combining (3.24) with the remaining calculations:

(1) $\int \phi^{2} \mathrm{~d}\|V\|=\lim _{i \rightarrow \infty} \int \phi_{i}^{2} \mathrm{~d}\left\|V^{i}\right\|$

(2) $\int\left|\nabla_{V} \phi\right|^{2} \mathrm{~d}\|V\|=\lim _{i \rightarrow \infty} \int\left|\nabla \phi_{i}\right|^{2} \mathrm{~d}\left\|V^{i}\right\|$,

(3) $\int|A|^{2} \phi^{2} \mathrm{~d}\|V\| \leq \liminf _{i \rightarrow \infty} \int\left|A^{i}\right|^{2} \phi_{i}^{2} \mathrm{~d}\left\|V^{i}\right\|$,

(4) $\int \operatorname{Ric}_{M}(\nu, \nu) \phi^{2} \mathrm{~d}\|V\|=\lim _{i \rightarrow \infty} \int \operatorname{Ric}_{M}\left(\nu_{i}, \nu_{i}\right) \phi_{i}^{2} \mathrm{~d}\left\|V^{i}\right\|$.

(1) By assumption $\phi_{i}^{2} \rightarrow \phi^{2}$ in $C_{c}(W)$, whence by Corollary A.3 we get $\int \phi^{2} \mathrm{~d}\|V\|=\lim _{i \rightarrow \infty} \int \phi_{i}^{2} \mathrm{~d}\left\|V^{i}\right\|$. The same argument proves (2), after noticing that $\left\langle\nabla \phi_{i}, \nabla d_{V}\right\rangle \equiv 0$ implies $|\nabla \phi|^{2}=\left|\nabla_{V} \phi\right|^{2}$ on $W \cap \operatorname{reg} V$.

(3) The sequence $\left(A^{i} \phi_{i} \mathrm{~d}\left\|V^{i}\right\|\right)$ converges weakly to $A \phi \mathrm{d}\|V\|$, as we can show by testing against an arbitrary $\varphi \in C_{c}(U)$ :

$$
\begin{aligned}
\int A^{i} \phi_{i} \varphi \mathrm{d}\left\|V^{i}\right\|-\int A \phi \varphi \mathrm{d}\|V\| & = \\
\int A^{i}\left(\phi_{i}-\phi\right) \varphi \mathrm{d}\left\|V^{i}\right\| & +\int A^{i} \phi \varphi \mathrm{d}\left\|V^{i}\right\|-\int A \phi \varphi \mathrm{d}\|V\| .
\end{aligned}
$$

The first integral is bounded by

$$
\left|\int A^{i}\left(\phi_{i}-\phi\right) \varphi \mathrm{d}\left\|V^{i}\right\|\right| \leq\left\|\phi_{i}-\phi\right\|_{L^{\infty}}\|\varphi\|_{L^{2}\left(V^{i}\right)}\left\|A^{i}\right\|_{L^{2}\left(V^{i}\right)} \rightarrow 0
$$

because $\phi_{i} \rightarrow \phi$ in $C_{c}(W)$ as $i \rightarrow \infty$. The remaining terms tend to 0 by the weak convergence of $A^{i} \mathrm{~d}\left\|V^{i}\right\| \rightarrow A \mathrm{~d}\|V\|$ tested against $\phi \varphi \in C_{c}(W)$. Then inequality (A.4) gives $\int|A|^{2} \phi^{2} \mathrm{~d}\|V\| \leq \liminf _{i \rightarrow \infty} \int\left|A^{i}\right|^{2} \phi_{i}^{2} \mathrm{~d}\left\|V^{i}\right\|$.

(4) For each $S \in G_{n}\left(T_{p} M\right)$ pick a unit vector $\nu_{S}$ in $T_{p} M$ orthogonal to $S$, and define a smooth function $R_{M}$ on $G_{n}(U)$ by $R_{M}: S \mapsto \operatorname{Ric}_{M}\left(\nu_{S}, \nu_{S}\right)$. Then $\phi_{i}^{2} R_{M} \rightarrow \phi^{2} R_{M}$ in $C_{c}\left(G_{n}(U)\right)$ as $i \rightarrow \infty$, and by Corollary A.3,

$$
\begin{aligned}
& \int \phi_{i}^{2} \operatorname{Ric}_{M}\left(\nu_{i}, \nu_{i}\right) \mathrm{d}\left\|V^{i}\right\|= \\
& \int \phi_{i}^{2} R_{M} \mathrm{~d} V^{i} \rightarrow \int \phi^{2} R_{M} \mathrm{~d} V=\int \phi^{2} \operatorname{Ric}_{M}(\nu, \nu) \mathrm{d}\|V\| .
\end{aligned}
$$

We conclude the section with a proof of Theorem $\mathrm{A}(\mathrm{i})$ in the case where there is a uniform $L^{2}\left(V^{i}\right)$-bound on the second fundamental forms $\left(A^{i}\right)$.

Lemma 3.12. Let $W \subset \subset U \backslash \operatorname{sing} V$ be open with $W \cap \operatorname{reg} V \neq \emptyset$. If $\sup _{i} \int_{W}\left|A^{i}\right|^{2} \mathrm{~d}\left\|V^{i}\right\|<+\infty$, then $\lambda_{p}(W) \geq \lim \sup _{i \rightarrow \infty} \lambda_{p}^{i}(W)$ for all $p$. 
Proof. We may assume that every connected component of $W$ intersects $\operatorname{spt}\|V\|$ (or $\operatorname{reg} V$, equivalently as $W \cap \operatorname{sing} V=\emptyset$ ) without restricting generality: if $C$ is a connected component of $W$ with $C \cap \operatorname{reg} V=\emptyset$, then $\lambda_{p}(W \backslash C)=\lambda_{p}(W)$, and by monotonicity $\lambda_{p}^{i}(W \backslash C) \geq \lambda_{p}^{i}(W)$ for all $i$.

Given $\delta>0$ there is a $p$-dimensional linear subspace $S=\operatorname{span}\left(\phi_{1}, \ldots, \phi_{p}\right)$ of $C_{c}^{1}(W \cap \operatorname{reg} V)$ with

$$
\lambda_{p}(W)+\delta \geq \max _{S \backslash\{0\}} J_{V}
$$

Extend the functions $\phi_{i}$ to $C_{c}^{1}(W)$ as in Lemma 3.10; for large enough $i$ the family $\left(\left|\nabla u_{i}\right| \phi_{1}, \ldots,\left|\nabla u_{i}\right| \phi_{p}\right)$ is still linearly independent. Indeed, otherwise we could extract a subsequence such that $\left(\left|\nabla u_{i^{\prime}}\right| \phi_{1}, \ldots,\left|\nabla u_{i^{\prime}}\right| \phi_{p}\right)$ has a linear dependence, with coefficients $a_{i^{\prime}} \neq 0 \in \mathbb{R}^{p}$ say. Then notice that

$$
\left|\nabla u_{i^{\prime}}\right| a_{i^{\prime}} \cdot \phi=0 \Leftrightarrow\left\|a_{i^{\prime}} \cdot \phi\right\|_{L^{2}\left(V^{i^{\prime}}\right)}=0,
$$

where we abbreviated $a_{i^{\prime}} \cdot \phi:=\sum_{j=1}^{p} a_{i^{\prime} j}\left|\nabla u_{i^{\prime}}\right| \phi_{j}$. We may normalise the coefficients $a_{i^{\prime}}$ so as to guarantee $\left|a_{i^{\prime}}\right|=1$ and then, possibly after extracting a second subsequence, assume that $a_{i^{\prime}} \rightarrow a \in \mathbb{S}^{p-1}$ as $i^{\prime} \rightarrow \infty$. The resulting strong convergence $a_{i^{\prime}} \cdot \phi \rightarrow a \cdot \phi$ in $C_{c}(W)$ combined with $\left\|V^{i}\right\| \rightarrow\|V\|$ yield $\left\|a_{i^{\prime}} \cdot \phi\right\|_{L^{2}\left(V^{i^{\prime}}\right)} \rightarrow\|a \cdot \phi\|_{L^{2}(V)}$; this contradicts $\|a \cdot \phi\|_{L^{2}(V)}>0$.

From now on take $i$ large enough so that $\left(\left|\nabla u_{i}\right| \phi_{1}, \cdots,\left|\nabla u_{i}\right| \phi_{p}\right)$ is linearly independent. For such large $i$, we may let $t_{i} \in \mathbb{S}^{p-1} \subset \mathbb{R}^{p}$ be the (normalised) coefficients of a linear combination $t_{i} \cdot\left|\nabla u_{i}\right| \phi=\sum_{j=1}^{p} t_{i j}\left|\nabla u_{i}\right| \phi_{j}$ that maximises the Rayleigh quotient $J_{i}$ :

$$
J_{i}\left(t_{i} \cdot\left|\nabla u_{i}\right| \phi\right)=\max _{\left|\nabla u_{i}\right| S \backslash\{0\}} J_{i} \geq \lambda_{p}^{i}(W) .
$$

Extract a convergent subsequence $t_{i^{\prime}} \rightarrow t \in \mathbb{S}^{p-1}$, so that $t_{i^{\prime}} \cdot \phi \rightarrow t \cdot \phi$ in $C_{c}^{1}(W)$ as $i^{\prime} \rightarrow \infty$. Lemma 3.11 gives $J_{V}(t \cdot \phi) \geq \lim \sup _{i \rightarrow \infty} \max _{\left|\nabla u_{i}\right| S \backslash\{0\}} J_{i}$, which in turn is greater than $\lim \sup _{i \rightarrow \infty} \lambda_{p}^{i}(W)$. Using (3.28),

$$
\lambda_{p}(W)+\delta \geq J_{V}(t \cdot \phi) \geq \limsup _{i \rightarrow \infty} \lambda_{p}^{i}(W),
$$

and we conclude by letting $\delta \rightarrow 0$.

Lemma 3.12 has the following immediate corollary.

Corollary 3.13. Under the hypotheses of Lemma 3.12,

$$
\operatorname{ind}_{W} B_{V} \leq \liminf _{i \rightarrow \infty}\left(\operatorname{ind}_{W} u_{i}\right) .
$$

\section{Proof of the main theorem (Theorem A)}

We briefly recall the context of the proof: $M^{n+1}$ is a closed Riemannian manifold and $U \subset M$ is an arbitrary open subset. The sequence of functions $\left(u_{i}\right)$ in $C^{3}(U)$ satisfies Hypotheses (A), (B) and (C) - the last hypothesis says that ind $u_{i} \leq k$ for all $i$. To every $u_{i}$ we associate the $n$-varifold $V^{i}$ from (2.16). By Theorem 2.7, we may pass to a subsequence of $\left(V^{i}\right)$ converging weakly to a stationary integral varifold $V$. 
4.1. Spectrum and index of $V$ : proof of (i) and (ii). The main idea, inspired by an argument of Bellettini-Wickramasekera [BW18], is to fix a compactly contained open subset $W \subset \subset U \backslash \operatorname{sing} V$ and study the stability of $u_{i}$ in open balls covering $W \cap \operatorname{reg} V \neq \emptyset$. We then shrink the radii of the covering balls to 0 , and prove the spectral lower bound of Theorem A(i) by induction on $k$. The upper bound on ind $B_{V}$ of Theorem A(ii) is then an immediate consequence.

In the base of induction the $u_{i}$ are stable in $U$. Let $\eta \in C_{c}^{1}(U)$ be a cutoff function constant equal to 1 on $W$. The stability inequality (3.8) gives that

$$
\int_{W}\left|A^{i}\right|^{2} \mathrm{~d}\left\|V^{i}\right\| \leq C(M) \operatorname{dist}(W, \partial U)^{-2}\left\|V^{i}\right\|(U) \text { for all } i .
$$

Combining this with (2.17) we get $\sup _{i} \int_{W}\left|A^{i}\right|^{2} \mathrm{~d}\left\|V^{i}\right\|<+\infty$, and thus $\lambda_{p}(W) \geq \lim \sup _{i \rightarrow \infty} \lambda_{p}^{i}(W)$ by Lemma 3.12 .

For the induction step, let $k \geq 1$ and assume that Theorem A(i) holds with $k-1$ in place of $k$. Consider an arbitrary $W \subset \subset U \backslash \operatorname{sing} V$ that intersects $\operatorname{reg} V$. Fix a radius $0<r<\operatorname{dist}(W, \operatorname{sing} V)$, and pick points $x_{1}, \ldots, x_{N} \in W \cap \operatorname{reg} V$ such that $W \cap \operatorname{reg} V \subset \cup_{j=1}^{N} B\left(x_{j}, \frac{r}{2}\right)$. We define the following Stability Condition for the cover $\left\{B\left(x_{j}, \frac{r}{2}\right)\right\}_{1 \leq j \leq N}$ :

(SC) For large $i$, each $u_{i}$ is stable in every ball $B\left(x_{1}, r\right), \ldots, B\left(x_{N}, r\right)$.

Claim 1. If for the cover $\left\{B\left(x_{j}, \frac{r}{2}\right)\right\}_{1 \leq j \leq N}$ :

(a) (SC) holds, then $\lambda_{p}(W) \geq \lim \sup _{i \rightarrow \infty} \lambda_{p}^{i}(W)$;

(b) (SC) fails, then $\lambda_{p}\left(W \backslash \overline{B_{r}}\right) \geq \lim \sup _{i \rightarrow \infty} \lambda_{p}^{i}\left(W \backslash \overline{B_{r}}\right)$ for some ball $B_{r} \in\left\{B\left(x_{j}, r\right)\right\}$.

Proof. (a) Let $W_{r}=W \cap \cup_{j=1}^{N} B\left(x_{j}, \frac{r}{2}\right)$, so that $W_{r} \cap \operatorname{reg} V=W \cap \operatorname{reg} V$ and hence $\lambda_{p}\left(W_{r}\right)=\lambda_{p}(W)$. Moreover $W_{r} \subset W$, so $\lambda_{p}^{i}\left(W_{r}\right) \geq \lambda_{p}^{i}(W)$ for all $i$ by monotonicity of the spectrum. Therefore it is enough to show that $\lambda_{p}\left(W_{r}\right) \geq \lim \sup _{i \rightarrow \infty} \lambda_{p}^{i}\left(W_{r}\right)$.

Because (SC) holds, summing (3.9) over all balls we get

$$
\int_{W_{r}}\left|A^{i}\right|^{2} \mathrm{~d}\left\|V^{i}\right\| \leq \frac{N C}{r^{2}}\left\|V^{i}\right\|\left(W_{r}\right) \leq \frac{N C E_{0}}{2 r^{2} \sigma} \text { for all } i,
$$

so $\lambda_{p}\left(W_{r}\right) \geq \lim \sup _{i \rightarrow \infty} \lambda_{p}^{i}\left(W_{r}\right)$ by Lemma 3.12 .

(b) If (SC) fails, then some subsequence $\left(u_{i^{\prime}}\right)$ must be unstable in one of the balls $B_{r} \in\left\{B\left(x_{j}, r\right)\right\}$, in other words $\operatorname{ind}_{B_{r}} u_{i^{\prime}} \geq 1$ for all $i^{\prime}$. On the other hand

$$
\operatorname{ind}_{B_{r}} u_{i^{\prime}}+\operatorname{ind}_{W \backslash \overline{B_{r}}} u_{i^{\prime}} \leq \operatorname{ind}_{W} u_{i^{\prime}}
$$

because $B_{r}$ and $W \backslash \overline{B_{r}}$ are disjoint open sets. As $\operatorname{ind}_{W} u_{i^{\prime}} \leq k$, we get $\operatorname{ind}_{W \backslash \overline{B_{r}}} u_{i^{\prime}} \leq k-1$ for all $i^{\prime}$, and we conclude after applying the induction hypothesis to $\left(u_{i^{\prime}}\right)$ in $W \backslash \overline{B_{r}}$.

Remark 4.1. This argument shows that when (SC) fails there is a ball $B_{r} \in$ $\left\{B\left(x_{j}, r\right)\right\}$ with $\lambda_{p}\left(W \backslash \overline{B_{r}}\right) \geq \limsup _{i \rightarrow \infty} \lambda_{p+1}^{i}(W)$ for $p \geq k$, and thus also ind $_{W \backslash \overline{B_{r}}} B_{V} \leq k-1$, but the induction step only requires the weaker conclusion from Claim 1. 
Now consider a decreasing sequence $r_{m} \rightarrow 0$ with $0<r_{m}<\operatorname{dist}(W, \operatorname{sing} V)$ and reason as above with $r=r_{m}$. For each $m$, pick points $x_{1}^{m}, \ldots, x_{N_{m}}^{m} \in$ $W \cap \operatorname{reg} V$ such that

$$
W \cap \operatorname{reg} V \subset \cup_{j=1}^{N_{m}} B\left(x_{j}^{m}, \frac{r_{m}}{2}\right) .
$$

If (SC) holds for a cover $\left\{B\left(x_{j}^{m}, \frac{r_{m}}{2}\right)\right\}$, then $\lambda_{p}(W) \geq \lim \sup _{i \rightarrow \infty} \lambda_{p}^{i}(W)$ by Claim 1, and the induction step is completed.

Otherwise (SC) fails for all constructed covers, and by Claim 1 there is a sequence $\left(y_{m}\right)$ in $W \cap \operatorname{reg} V$ with

$$
\lambda_{p}\left(W \backslash \bar{B}\left(y_{m}, r_{m}\right)\right) \geq \limsup _{i \rightarrow \infty} \lambda_{p}^{i}\left(W \backslash \bar{B}\left(y_{m}, r_{m}\right)\right),
$$

and thus by monotonicity of the spectrum

$$
\lambda_{p}\left(W \backslash \bar{B}\left(y_{m}, r_{m}\right)\right) \geq \limsup _{i \rightarrow \infty} \lambda_{p}^{i}(W) .
$$

Passing to a subsequence if necessary, we may assume that $\left(y_{m}\right)$ converges to a point $y \in \bar{W} \cap \operatorname{reg} V$. If we fix a radius $R>0$, then $B\left(y_{m}, r_{m}\right) \subset B(y, R)$ for large enough $m$, so by monotonicity and (4.6),

$$
\lambda_{p}(W \backslash \bar{B}(y, R)) \geq \limsup _{m \rightarrow \infty} \lambda_{p}\left(W \backslash \bar{B}\left(y_{m}, r_{m}\right)\right) \geq \limsup _{i \rightarrow \infty} \lambda_{p}^{i}(W) .
$$

The conclusion follows after combining this with $\lambda_{p}(W)=\lim _{R \rightarrow 0} \lambda_{p}(W \backslash$ $\bar{B}(y, R))$ from Lemma 3.7 .

Together with the base of induction, we have proved Theorem A(i) for all sequences $\left(u_{i}\right)$ with $\sup _{i}$ ind $u_{i} \leq k$ for some $k \in \mathbb{N}$. The index bound ind $_{W} B_{V} \leq k$ follows immediately: the spectral lower bound implies that $L_{V}$ must have fewer negative eigenvalues than the $L_{i}$ as $i \rightarrow \infty$. Therefore

$$
\operatorname{ind}_{W} B_{V}=\operatorname{card}\left\{p \in \mathbb{N} \mid \lambda_{p}(W)<0\right\} \leq k .
$$

As the subset $W$ was arbitrary we get ind $B_{V} \leq k$; this proves Theorem A(ii).

4.2. Regularity of $V$ : proof of $\operatorname{dim}_{\mathcal{H}} \operatorname{sing} V \leq n-7$. The approach is the same as in the proof of Theorem A(i)-(ii) with one difference: we again proceed by induction on $k$, but we now cover the entire support $\operatorname{spt}\|V\|$ (including the singular set), instead of constructing covers a positive distance away from $\operatorname{sing} V$.

The base of induction, where the $u_{i}$ are stable in $U$, was proved in [TW12].

For the induction step, suppose that $\operatorname{dim}_{\mathcal{H}}\left(U^{\prime} \cap \operatorname{sing} V\right) \leq n-7$ holds with $k-1$ in place of $k$, and for arbitrary open subsets $U^{\prime} \subset U$. Fix $r>0$, and choose points $x_{1}, \ldots, x_{N} \in U \cap \operatorname{spt}\|V\|$ such that $U \cap \operatorname{spt}\|V\| \subset \cup_{j=1}^{N} B\left(x_{j}, r\right)$. The Stability Condition for the cover $\left\{B\left(x_{j}, r\right)\right\}_{1 \leq j \leq N}$ is defined in the same way as above, except the radii need not be doubled:

(SC) For large $i$, each $u_{i}$ is stable in every ball $B\left(x_{1}, r\right), \ldots, B\left(x_{N}, r\right)$.

Claim 2. If for the cover $\left\{B\left(x_{j}, r\right)\right\}_{1 \leq j \leq N}$ :

(a) (SC) holds, then $\operatorname{dim}_{\mathcal{H}} \operatorname{sing} V \leq n-7$;

(b) (SC) fails, then $\operatorname{dim}_{\mathcal{H}} \operatorname{sing} V \backslash \overline{B_{r}} \leq n-7$ for some ball $B_{r} \in$ $\left\{B\left(x_{j}, r\right)\right\}$. 
Proof. (a) The results from [TW12] give $\operatorname{dim}_{\mathcal{H}} B\left(x_{j}, r\right) \cap \operatorname{sing} V \leq n-7$ for every $j=1, \ldots, N$. As the balls $\left\{B\left(x_{j}, r\right)\right\}$ cover $U \cap \operatorname{spt}\|V\|$, the same holds for $\operatorname{sing} V$.

(b) When (SC) fails, there must be a subsequence $\left(u_{i^{\prime}}\right)$ that is unstable in one of the balls $B_{r}$ of the cover, so that in its complement

$$
\operatorname{ind}_{U \backslash \overline{B_{r}}} u_{i^{\prime}} \leq k-1 \text { for all } i^{\prime} .
$$

The conclusion follows from the induction hypothesis applied to $\left(u_{i^{\prime}}\right)$ in $U \backslash \overline{B_{r}}$.

Now consider a decreasing sequence $r_{m} \rightarrow 0$. For every $m$, choose points $x_{1}^{m}, \ldots, x_{N_{m}}^{m} \in U \cap \operatorname{spt}\|V\|$ such that $U \cap \operatorname{spt}\|V\| \subset \cup_{j=1}^{N_{m}} B\left(x_{j}^{m}, r_{m}\right)$. Then either (SC) holds for the cover $\left\{B\left(x_{j}^{m}, r_{m}\right)\right\}$ constructed for some $m$, in which case we can conclude from Claim 2, or else there is sequence of points $\left(y_{m}\right)$ in $U \cap \operatorname{spt}\|V\|$ for which

$$
\operatorname{dim}_{\mathcal{H}} \operatorname{sing} V \backslash \bar{B}\left(y_{m}, r_{m}\right) \leq n-7 .
$$

Possibly after extracting a subsequence, the sequence $\left(y_{m}\right)$ converges to a point $y \in \bar{U} \cap \operatorname{spt}\|V\|$. As $U \backslash\{y\} \subset \cup_{m \geq 0} U \backslash \bar{B}\left(y_{m}, r_{m}\right)$, we get $\operatorname{dim}_{\mathcal{H}}(\operatorname{sing} V \backslash\{y\}) \leq n-7$.

If $n \geq 7$, then $\operatorname{dim}_{\mathcal{H}} \operatorname{sing} V \leq n-7$ holds whether or not $y \in \operatorname{sing} V$, as points are zero-dimensional. If however $2 \leq n \leq 6$ then we need $\operatorname{sing} V=\emptyset$, which amounts to the following claim.

Claim 3. If $2 \leq n \leq 6$ then $y \notin \operatorname{sing} V$.

Proof. Choose $B(y, R) \subset U$, and consider balls $\left\{B\left(y, R_{m}\right)\right\}_{m \in \mathbb{N}}$ with shrinking radii $R_{m}:=2^{-m} R$. If for some $m$ there is a subsequence $\left(u_{i^{\prime}}\right)$ with

$$
\operatorname{ind}_{B\left(y, R_{m}\right)} u_{i^{\prime}} \leq k-1 \text { for all } i^{\prime},
$$

then we can conclude from the induction hypothesis. Otherwise for all $m$

$$
\operatorname{ind}_{B\left(y, R_{m}\right)} u_{i}=k \text { for } i \text { large enough, }
$$

and the $u_{i}$ are eventually stable in the annulus $B(y, R) \backslash \bar{B}\left(y, R_{m}\right)$. By Theorem A(ii)

$$
\operatorname{ind}_{B(y, R) \backslash \bar{B}\left(y, R_{m}\right)} B_{V}=0 \text { for all } R_{m} \rightarrow 0,
$$

and thus ind $\operatorname{in}_{B(y, R) \backslash\{y\}} B_{V}=0$.

By contradiction, suppose that $y \in \operatorname{sing} V$. Then ind ${ }_{B(y, r)} B_{V}=0$ holds in the whole ball $B(y, r)$ away from $\operatorname{sing} V$, and the regularity results of [Wic14] give $\operatorname{dim}_{\mathcal{H}} B(y, R) \cap \operatorname{sing} V \leq n-7$, so that $y \notin \operatorname{sing} V$.

Claim 3 concludes the induction step; together with the base of induction, we have proved that $\operatorname{dim}_{\mathcal{H}} \operatorname{sing} V \leq n-7$. This finishes the proof of Theorem A. 


\section{Appendix A. Measure-Function convergence}

In this appendix we give two elementary measure-theoretical lemmas that are used in the proofs of Lemma 3.11 and in Appendix B. Essentially they give a weak compactness result for sequences of the form $\left(f_{i} \mathrm{~d} \mu_{i}\right)_{i \in \mathbb{N}}$, with $\mu_{i}$ Radon measures and $f_{i} \in L^{2}\left(\mu_{i}\right)$. The weak convergence in question is sometimes called measure-function convergence in the literature. It appears in the work of Hutchinson [Hut86] on so-called curvature varifolds; there one also finds a proof of Lemma A.1 under more general hypotheses on the sequence $\left(f_{i}\right)$.

Lemma A.1 ([Hut86; Ton05]). Let $X$ be a locally compact Hausdorff space, let $\left(\mu_{i}\right)_{i \in \mathbb{N}}$ be a sequence of Radon measures on $X$, and $\left(f_{i}: X \rightarrow \mathbb{R}\right)_{i \in \mathbb{N}}$ be a sequence of Borel-measurable functions. Suppose that

$$
\begin{array}{r}
\sup _{i} \mu_{i}(X)<+\infty, \\
\sup _{i} \int_{X} f_{i}^{2} \mathrm{~d} \mu_{i}<+\infty .
\end{array}
$$

Then there is a Radon measure $\mu$ and $f \in L^{2}(\mu)$ such that for some subsequence $\mu_{i^{\prime}} \rightarrow \mu$ and $f_{i^{\prime}} \mathrm{d} \mu_{i^{\prime}} \rightarrow f \mathrm{~d} \mu$ weakly as Radon measures, i.e.

$$
\int_{X} f_{i^{\prime}} \phi \mathrm{d} \mu_{i^{\prime}} \rightarrow \int_{X} f \phi \mathrm{d} \mu \quad \text { for all } \phi \in C_{c}(X) .
$$

Moreover, the weak limit $f \mathrm{~d} \mu$ satisfies

$$
\int_{X} f^{2} \mathrm{~d} \mu \leq \liminf _{i \rightarrow \infty} \int_{X} f_{i}^{2} \mathrm{~d} \mu_{i}
$$

Remark A.2. In our applications $X$ is either an open subset of $U \subset M$ or its Grassmannian $G_{n}(U)$, and $\mu_{i}$ is either $\left\|V^{i}\right\|$ or $V^{i}$.

Proof. The signed Radon measures $\nu_{i}:=f_{i} \mathrm{~d} \mu_{i}$ have bounded total variation, so that the sequences $\left(\mu_{i}\right)$ and $\left(\nu_{i}\right)$ have convergent subsequences, with limits the Radon measures $\mu$ and $\nu$ respectively. We extract these subsequences without relabelling their indices.

Consider an arbitrary $\phi \in C_{c}(X)$. By the weak convergence $\nu_{i} \rightarrow \nu$,

$$
\int \phi \mathrm{d} \nu=\lim _{i \rightarrow \infty} \int \phi f_{i} \mathrm{~d} \mu_{i} \leq\|\phi\|_{L^{2}(\mu)} \liminf _{i \rightarrow \infty}\left\|f_{i}\right\|_{L^{2}\left(\mu_{i}\right)},
$$

where we used the weak convergence $\mu_{i} \rightarrow \mu$ to get $\lim _{i \rightarrow \infty}\|\phi\|_{L^{2}\left(\mu_{i}\right)}=$ $\|\phi\|_{L^{2}(\mu)}$. As $C_{c}(X)$ is dense in $L^{2}(\mu)$, the measure $\nu$ defines a bounded linear functional on $L^{2}(\mu)$, and by duality there is $f \in L^{2}(\mu)$ with $\|f\|_{L^{2}(\mu)} \leq$ $\liminf _{i \rightarrow \infty}\left\|f_{i}\right\|_{L^{2}\left(\mu_{i}\right)}$ such that $\nu=f \mathrm{~d} \mu$.

If the densities $f_{i}$ are in $C_{c}(X)$ and converge strongly, then their limit coincides with the density of the weak limit of $\left(f_{i} \mathrm{~d} \mu_{i}\right)$.

Corollary A.3. Additionally to the hypotheses of Lemma A.1, assume that $f_{i} \in C_{c}(X)$, and that $\left\|f_{i}-f\right\|_{L^{\infty}} \rightarrow 0$ for some $f \in C_{c}(X)$. Then, additionally to the conclusions of Lemma A.1,

$$
\int_{X} f^{2} \mathrm{~d} \mu=\lim _{i \rightarrow \infty} \int_{X} f_{i}^{2} \mathrm{~d} \mu_{i}
$$


Proof. We first show that $f_{i} \mathrm{~d} \mu_{i} \rightarrow f \mathrm{~d} \mu$. Let $\varphi \in C_{c}(X)$ be arbitrary, then

$$
\int f_{i} \varphi \mathrm{d} \mu_{i}-\int f \varphi \mathrm{d} \mu=\int\left(f_{i}-f\right) \varphi \mathrm{d} \mu_{i}+\int f \varphi \mathrm{d} \mu_{i}-\int f \varphi \mathrm{d} \mu .
$$

The first term $\left|\int\left(f_{i}-f\right) \varphi \mathrm{d} \mu_{i}\right| \leq\left\|f_{i}-f\right\|_{L^{\infty}}\|\varphi\|_{L^{1}\left(\mu_{i}\right)} \rightarrow 0$ as $i \rightarrow \infty$. The remaining terms $\int f \varphi \mathrm{d} \mu_{i}-\int f \varphi \mathrm{d} \mu \rightarrow 0$ as $i \rightarrow \infty$ by the weak convergence $\mu_{i} \rightarrow \mu$. We reason similarly to show (A.6):

$$
\left|\int_{X} f^{2} \mathrm{~d} \mu-\int_{X} f_{i}^{2} \mathrm{~d} \mu_{i}\right| \leq\left|\int_{X} f^{2} \mathrm{~d} \mu-\int_{X} f^{2} \mathrm{~d} \mu_{i}\right|+\mu_{i}(X)\left\|f_{i}^{2}-f^{2}\right\|_{L^{\infty}} .
$$

The first term goes to 0 by the weak convergence $\mu_{i} \rightarrow \mu$, and so does the second $\operatorname{assup}_{i} \mu_{i}(X)<+\infty$ and $\left\|f_{i}^{2}-f^{2}\right\|_{L^{\infty}} \rightarrow 0$.

\section{Appendix B. Generalised second fundamental forms}

Our main aim in this appendix is to give a proof of Proposition 3.6. We follow the approach of [Ton05], where the case of stable $u_{i}$ is treated using notions from [Hut86]. Our account is self-contained but for the fact that we refer to these two works for some technical, but routine calculations.

Throughout this section, we assume that $U \subset M$ is isometrically embedded in some $\mathbb{R}^{q}$, and $W \subset \subset U \backslash \operatorname{sing} V$ is an open subset with $W \cap \operatorname{reg} V \neq \emptyset$. The fibre of the Grassmannian $G_{n}(U)$ at $x \in U$ is identified with the subspaces

$$
\left\{S \subset \mathbb{R}^{q} \mid S \subset T_{x} M, \operatorname{dim} S=n\right\} \subset U \times G(n, q),
$$

where $G(n, q)=\left\{S \subset \mathbb{R}^{q} \mid \operatorname{dim} S=n\right\}$. We furthermore identify an element $S \in U \times G(n, q)$ with the corresponding orthogonal projection $\mathbb{R}^{q} \rightarrow S$, so that $G_{n}(U) \subset U \times \mathbb{R}^{q^{2}}$. Throughout, $P(x) \in \mathbb{R}^{q^{2}}$ represents the orthogonal projection $\mathbb{R}^{q} \rightarrow T_{x} M$ and $\left(e_{1}, \ldots, e_{q}\right)$ is the standard basis of $\mathbb{R}^{q} ; \partial_{i}$ and $\partial_{i j}^{*}$ denote differentiation with respect to $e_{i}$ and $e_{i} \otimes e_{j} \in \mathbb{R}^{q^{2}}$ respectively.

Consider first a smooth embedded hypersurface $\Sigma \subset U$, which we implicitely identify with the varifold $V_{\Sigma}:=V_{\Sigma, 1}$ with constant multiplicity. We consider test functions $\phi \in C^{1}\left(U \times \mathbb{R}^{q^{2}}\right)$ with compact spatial support, that is with compact support in the first variable. We associate to it a function $\varphi \in C_{c}^{1}(\Sigma)$ defined by $\varphi(x)=\phi\left(x, S^{\Sigma}(x)\right)$, where $S^{\Sigma}(x) \in \mathbb{R}^{q^{2}}$ is the orthogonal projection $\mathbb{R}^{q} \rightarrow T_{x} \Sigma$. Define a vector field $X \in C_{c}^{1}(\Sigma, T M)$ by $X=\varphi P\left(e_{j}\right)$, where $e_{j}$ is one of the standard basis vectors. Its component tangential to $\Sigma$ is $\varphi S^{\Sigma}\left(e_{j}\right)$, and by the standard divergence theorem we get $\int_{\Sigma} \operatorname{div}_{\Sigma}\left(\varphi S^{\Sigma} e_{j}\right)=0$. A routine calculation shows that in coordinates

$$
\operatorname{div}_{\Sigma}\left(\varphi S^{\Sigma} e_{j}\right)=S_{r j}^{\Sigma} \partial_{r} \phi+\phi S_{r i}^{\Sigma} \partial_{i} S_{j r}^{\Sigma}+S_{j i}^{\Sigma} \partial_{i} S_{k r}^{\Sigma} \partial_{k r}^{*} \phi
$$

with summation over repeated indices [Hut86]. Abbreviate $B_{j k r}^{\Sigma}=S_{j i}^{\Sigma} \partial_{i} S_{k r}^{\Sigma}$ and substitute this into the divergence formula:

$$
\begin{aligned}
0 & =\int_{\Sigma} S_{r j}^{\Sigma} \partial_{r} \phi+B_{r j r}^{\Sigma} \phi+B_{j k r}^{\Sigma} \partial_{k r}^{*} \phi \mathrm{d} \mathcal{H}^{n} \\
& =\int_{G_{n}(U)} S_{r j} \partial_{r} \phi+B_{r j r}^{\Sigma} \phi+B_{j k r}^{\Sigma} \partial_{k r}^{*} \phi \mathrm{d} V_{\Sigma}(x, S) .
\end{aligned}
$$

This identity is the basis of the following definition by Hutchinson [Hut86]. 
Definition B.1 (Generalised curvature, [Hut86]). An $n$-dimensional integral varifold $V$ in $U$ is said to have generalised curvature if there exists a function $B=\left(B_{i j k}\right)$ with values in $\mathbb{R}^{q^{3}}$ defined $V$-a.e. on $G_{n}(U)$ with

(a) $B \in L_{\text {loc }}^{1}(V)$,

(b) $\int_{G_{n}(U)} S_{r j} \partial_{r} \phi+B_{r j r} \phi+B_{j k r} \partial_{k r}^{*} \phi \mathrm{d} V(x, S)=0$ for all $\phi \in C^{1}\left(U \times \mathbb{R}^{q^{2}}\right)$ with compact spatial support.

The following lemma shows that the function $B$ is well-defined $V$-a.e. on $G_{n}(U)$; it is taken from [Hut86].

Lemma B.2 ([Hut86]). Any two $B$ and $\widetilde{B}$ satisfying (a) and (b) coincide $V$-a.e. on $G_{n}(U)$.

Proof. Let $\phi(x, S)=\alpha(x) \beta(S)$, where $\alpha \in C_{c}^{1}(U)$ and $\beta \in C^{1}\left(\mathbb{R}^{q^{2}}\right)$. Letting $\beta \equiv 1$ we see that $\int B_{r j r} \alpha \mathrm{d} V=\int \widetilde{B}_{r j r} \alpha \mathrm{d} V$, and thus $B_{r j r}=\widetilde{B}_{r j r} V$-a.e. on $G_{n}(U)$. If we now let

$$
\beta(S)= \begin{cases}1 & \text { if } S=S_{k r} \\ 0 & \text { otherwise }\end{cases}
$$

then $\int B_{j k r} \alpha \mathrm{d} V=\int \widetilde{B}_{j k r} \alpha \mathrm{d} V$, whence the conclusion follows.

In particular, applied to the smooth hypersurfaces, the following is an immediate consequence.

Corollary B.3. If $\Sigma$ is a smoothly embedded hypersurface, then $B_{i j k}(x, S)=$ $B_{i j k}^{\Sigma}(x, S)$ for $V_{\Sigma}$-a.e. $(x, S) \in G_{n}(U)$, where $B_{i j k}^{\Sigma}(x, S)=S_{i l} \partial_{l} S_{j k}^{\Sigma}$.

The following elementary calculation relates $B^{\Sigma}$ to the second fundamental form $A^{\Sigma}$.

Lemma B.4. Let $A^{\Sigma}$ be the second fundamental form of a smoothly embedded hypersurface $\Sigma \subset U$. Then

$$
\left\langle A^{\Sigma}\left(S^{\Sigma} e_{i}, S^{\Sigma} e_{j}\right), P e_{k}\right\rangle=P_{k r} S_{j s}^{\Sigma} S_{i l}^{\Sigma} \partial_{l} S_{r s}^{\Sigma}=P_{k r} S_{j s}^{\Sigma} B_{i r s}^{\Sigma}\left(x, S^{\Sigma}\right) .
$$

Proof. Write $A$ instead of $A^{\Sigma}$ in this proof to simplify notation. The covariant derivative on $M$ is the component of $D=\nabla^{\mathbb{R}^{q}}$ tangent to $M$, so $A=\left(D^{T_{M}}\right)^{\perp_{\Sigma}}=\left(D^{\perp_{\Sigma}}\right)^{T_{M}}$. As $e_{k}^{T_{M}}=P_{k r} e_{r}$, we get

$$
\begin{aligned}
A_{i j}^{k} & :=\left\langle A\left(S^{\Sigma} e_{i}, S^{\Sigma} e_{j}\right), e_{k}^{T_{M}}\right\rangle \\
& =\left\langle\left(D_{S^{\Sigma}} e_{i} S^{\Sigma} e_{j}\right)^{\perp_{\Sigma}}, P e_{k}\right\rangle=P_{k r}\left\langle D_{S^{\Sigma} e_{i}} S^{\Sigma} e_{j}, e_{r}^{\perp_{\Sigma}}\right\rangle .
\end{aligned}
$$

Similarly $e_{r}^{\perp}=\left(\delta_{r s}-S_{r s}^{\Sigma}\right) e_{s}$, so

$$
A_{i j}^{k}=P_{k r}\left(\delta_{r s}-S_{r s}^{\Sigma}\right)\left\langle D_{S^{\Sigma} e_{i}} S^{\Sigma} e_{j}, e_{s}\right\rangle=P_{k r}\left(\delta_{r s}-S_{r s}^{\Sigma}\right) D_{S^{\Sigma} e_{i}} S_{j s}^{\Sigma} .
$$

As $S_{r s}^{\Sigma} S_{j s}^{\Sigma}=S_{r j}^{\Sigma}$, we finally get $A_{i j}^{k}=P_{k r} S_{j s}^{\Sigma} D_{S^{\Sigma}} e_{i} S_{r s}^{\Sigma}=P_{k r} S_{j s}^{\Sigma} S_{i l}^{\Sigma} \partial_{l} S_{r s}^{\Sigma}$, as required.

This expression can then be used to generalise second fundamental forms from the smooth to the varifold setting. 
Definition B.5 (Generalised second fundamental forms, [Hut86]). Let $V$ be an integral $n$-varifold with generalised curvature. Then its generalised second fundamental form is the function $A=\left(A_{i j}^{k}\right)$ with values in $\mathbb{R}^{q^{3}}$ and defined at $V$-a.e. $(x, S) \in G_{n}(U)$ by

$$
A_{i j}^{k}(x, S)=P_{k r} S_{j s} B_{i r s} .
$$

For a smoothly embedded $\Sigma \subset U$, we see after combining Corollary B.3 with Lemma B.4 that the generalised second fundamental form $A$ of $V_{\Sigma}$ is equal to the classical second fundamental form $A^{\Sigma}$ in the sense that

$$
A_{i j}^{k}(x, S)=\left\langle A^{\Sigma}\left(S e_{i}, S e_{j}\right), P e_{k}\right\rangle \text { for } V_{\Sigma^{-a}} \text {.e. }(x, S) \in G_{n}(U) .
$$

We now want to relate these notions to the varifolds $V^{i}$ defined in the main body. To simplify notation, we fix a critical point $u=u_{i}$ with associated varifold $V^{\epsilon}=V^{i}$. We define a 'second fundamental form' for $V^{\epsilon}$ using the coordinate expressions from Lemma B.4.

Definition B.6. Define the functions $A^{\epsilon}=\left(A_{i j}^{\epsilon, k}\right)$ and $B^{\epsilon}=\left(B_{i j k}^{\epsilon}\right)$ with values in $\mathbb{R}^{q^{3}}$ at all $(x, S) \in G_{n}(U)$ where $\nabla u(x) \neq 0$ by

$$
\begin{aligned}
& B_{i j k}^{\epsilon}(x, S)=S_{i l} \partial_{l} S_{j k}^{\epsilon}, \\
& A_{i j}^{k, \epsilon}(x, S)=P_{k r} S_{j s} S_{i l} \partial_{l} S_{r s}^{\epsilon}=P_{k r} S_{j s} B_{i r s}^{\epsilon},
\end{aligned}
$$

where $S^{\epsilon}=S^{\epsilon}(x) \in \mathbb{R}^{q^{2}}$ represents the projection $\mathbb{R}^{q} \rightarrow T_{x}\{u=u(x)\}$, and $P=P(x) \in \mathbb{R}^{q^{2}}$ the projection $\mathbb{R}^{q} \rightarrow T_{x} M$.

Technically speaking the function $A^{\epsilon}$ is not the second fundamental form of $V^{\epsilon}$, as $B^{\epsilon}$ satisfies the integral identity of Definition B.1 only up to a small error term. This can be seen as follows: take an arbitrary vector field $X \in C_{c}^{1}(U, T M)$, multiply the Allen-Cahn equation by $\langle X, \nabla u\rangle$ and integrate by parts twice to obtain

$$
\int_{U}|\nabla u|^{2} \operatorname{div} X-\left\langle\nabla_{\nabla u} X, \nabla u\right\rangle=\int_{U}\left(\frac{|\nabla u|^{2}}{2}-\frac{W(u)}{\epsilon^{2}}\right) \operatorname{div} X,
$$

which using integration with respect to $V^{\epsilon}$ is equivalent to

$$
\int_{G_{n}(U)} \operatorname{div}_{S} X \mathrm{~d} V^{\epsilon}(x, S)=\frac{1}{2 \sigma} \int_{U}\left(\epsilon \frac{|\nabla u|^{2}}{2}-\frac{W(u)}{\epsilon}\right) \operatorname{div} X,
$$

where $\operatorname{div}_{S} X=\sum_{i=1}^{q}\left\langle D_{S e_{i}} X, S e_{i}\right\rangle$. As before let $X=\phi\left(x, S^{\epsilon}\right) S^{\epsilon}\left(e_{j}\right)$, where $\phi \in C^{1}\left(U \times \mathbb{R}^{q^{2}}\right)$ has compact spatial support. Substitute this into (B.14) and perform routine coordinate computations as before in (B.2) to get

$$
\begin{aligned}
& \int_{G_{n}(U)} S_{r j} \partial_{r} \phi+B_{r j r}^{\epsilon} \phi+B_{j k r}^{\epsilon} \partial_{k r}^{*} \phi \mathrm{d} V^{\epsilon}(x, S)= \\
& \frac{1}{2 \sigma} \int_{U}\left(\epsilon \frac{|\nabla u|^{2}}{2}-\frac{W(u)}{\epsilon}\right) \operatorname{div} X .
\end{aligned}
$$

The integral on the right-hand side goes to 0 as $\epsilon \rightarrow 0$ - this is (2.18) in Theorem 2.7. This justifies the abuse of language that is calling $A^{\epsilon}$ the 'second fundamental form' of $V^{\epsilon}$.

We now compare $A^{\epsilon}$ with the second fundamental form $A^{\Sigma}$ of the level sets of $u$ near regular points: take a point $x \in U$ with $\nabla u(x) \neq 0$. Then 
the level set $\{u=u(x)\}$ is embedded in a neighbourhood $B$ around $x$; write $\Sigma=\{u=u(x)\} \cap B$. The calculations from Lemma B.4 show that $A^{\epsilon}\left(x, S^{\epsilon}\right)=A^{\Sigma}(x)$, so the second fundamental forms from Definitions 3.1 and B.6 agree $V^{\epsilon}$-a.e. Combining this observation with (3.2), we get

$$
\left|A^{\epsilon}\right|^{2}\left(x, S^{\epsilon}\right)=\left|A^{\Sigma}\right|^{2}(x) \leq \frac{1}{|\nabla u|^{2}}\left(\left|\nabla^{2} u\right|^{2}-\left.|\nabla| \nabla u\right|^{2}\right) .
$$

Therefore, when $\delta^{2} E_{\epsilon}(u)(|\nabla u| \phi,|\nabla u| \phi) \geq 0$ for some $\phi \in C_{c}^{1}(U)$, then $\int_{G_{n}(U)}\left|A^{\epsilon}\right|^{2} \phi^{2} \mathrm{~d} V^{\epsilon} \leq \int_{U}|\nabla \phi|^{2}-\operatorname{Ric}\left(\nu^{\epsilon}, \nu^{\epsilon}\right) \phi^{2} \mathrm{~d}\left\|V^{\epsilon}\right\|$ as in (3.8), and Corollary 3.5 also remains valid.

All the results in this appendix were established for an arbitrary critical point $u \in C^{3}(U) \cap L^{\infty}(U)$, and thus are valid for every term in the sequence $\left(u_{i}\right)$ satisfying Hypotheses $(\mathrm{A})-(\mathrm{C})$. Let $\left(V^{i}\right)$ be the corresponding varifolds as in (2.16), and let $\left(A^{\epsilon_{i}}\right)$ be their second fundamental forms as in Definition B.6. We restate Proposition 3.6 in the following equivalent form, with $A^{\epsilon_{i}}$ in place of $A^{i}$.

Proposition B.7. If $\sup _{i} \int_{W}\left|A^{\epsilon_{i}}\right|^{2} \mathrm{~d}\left\|V^{i}\right\|<+\infty$, then some subsequence $A^{\epsilon_{i^{\prime}}} \mathrm{d} V^{i^{\prime}} \rightarrow A \mathrm{~d} V$ weakly as Radon measures on $G_{n}(W)$, and

$$
\int_{W}|A|^{2} \mathrm{~d}\|V\| \leq \liminf _{i \rightarrow \infty} \int_{W}\left|A^{\epsilon_{i}}\right|^{2} \mathrm{~d}\left\|V^{i}\right\|
$$

where $A$ is the classical second fundamental form of $\operatorname{reg} V \subset M$.

Proof. Routine calculations as above in the proof of Lemma B.4 show that $B^{\epsilon_{i}}$ is related to $A^{\epsilon_{i}}$ as follows for all $i$ :

$$
B_{j k l}^{\epsilon_{i}}\left(x, S^{\epsilon_{i}}\right)=A_{j k}^{l, \epsilon_{i}}+A_{j l}^{k, \epsilon_{i}}+S_{k s}^{\epsilon_{i}} S_{j r}^{\epsilon_{i}} \partial_{r} P_{s l}+S_{l s}^{\epsilon_{i}} S_{j r}^{\epsilon_{i}} \partial_{r} P_{k s} .
$$

If we square (B.18) and sum over $j, k, l=1, \ldots, q$, we get

$$
\left|B^{\epsilon_{i}}\right|^{2} \leq 8\left(\left|A^{\epsilon_{i}}\right|^{2}+|D P|^{2}\right) \quad V^{i} \text {-a.e. in } G_{n}(U)
$$

The term $|D P|^{2}:=\sum_{j, k, l}^{q}\left(\partial_{j} P_{k l}\right)^{2}$ can be bounded by some constant $C(M)$, so that $\sup _{i} \int_{W}\left|B^{\epsilon_{i}}\right|^{2} \mathrm{~d}\left\|V^{i}\right\|<+\infty$ as well.

By Lemma A.1 we can pass to convergent subsequences $A^{\epsilon_{i}} \mathrm{~d} V^{i} \rightarrow A \mathrm{~d} V$ and $B^{\epsilon_{i}} \mathrm{~d} V^{i} \rightarrow B \mathrm{~d} V$ with limits related by $A_{j k}^{l}=P_{l r} S_{k s} B_{j r s} V$-a.e. in $G_{n}(W)$. The limit $A \mathrm{~d} V$ also satisfies

$$
\int_{G_{n}(W)}|A|^{2} \mathrm{~d} V \leq \liminf _{i \rightarrow \infty} \int_{G_{n}(W)}\left|A^{\epsilon_{i}}\right|^{2} \mathrm{~d} V^{i}
$$

and the analogous inequality holds for $B \mathrm{~d} V$. Moreover the error term on the right-hand side of (B.15) tends to 0 as $i \rightarrow \infty$, so the weak limit $B \mathrm{~d} V$ satisfies

$$
\int_{G_{n}(W)} S_{r j} \partial_{r} \phi+B_{r j r} \phi+B_{j k r} \partial_{k r}^{*} \phi \mathrm{d} V(x, S)=0
$$

for all $\phi \in C^{1}\left(W \times \mathbb{R}^{q^{2}}\right)$ with compact spatial support. By Corollary B.3 we have $B=B^{\operatorname{reg} V}$ and thus also $A=A^{\text {reg } V} V$-a.e. in $G_{n}(W)$. This concludes the proof. 


\section{REFERENCES}

[ACS16] L. Ambrozio, A. Carlotto, and B. Sharp. "Compactness of the space of minimal hypersurfaces with bounded volume and $p$-th Jacobi eigenvalue". Journal of Geometric Analysis 26(4) (2016), pp. 2591-2601.

[Alm65] F. Almgren. The Theory of Varifolds: A Variational Calculus in the Large for the $k$-dimensional Area Integrand. Institute of Advanced Study, 1965.

[BW18] C. Bellettini and N. Wickramasekera. Stable CMC integral varifolds of codimension 1: regularity and compactness. arXiv:1802.00377 [math.DG]. 2018.

[CM11] T. Colding and W. Minicozzi. A Course in Minimal Surfaces. Graduate Studies in Mathematics. American Mathematical Society, 2011.

[CM18] O. Chodosh and C. Mantoulidis. Minimal surfaces and the AllenCahn equation on 3-manifolds: index, multiplicity, and curvature estimates. arXiv:2803.02716 [math.DG]. 2018.

[DT13] C. De Lellis and D. Tasnady. "The existence of embedded minimal hypersurfaces". Journal of Differential Geometry 95(3) (2013), pp. $355-388$.

[EG15] L. Evans and R. Gariepy. Measure Theory and Fine Properties of Functions, Revised Edition. Textbooks in Mathematics. CRC Press, 2015.

[FSV13] A. Farina, Y. Sire, and E. Valdinoci. "Stable solutions of elliptic equations on Riemannian manifolds". Journal of Geometric Analysis 23(3) (2013), pp. 1158-1172.

[Gas17] P. Gaspar. The second inner variation of energy and the Morse index of limit interfaces. arXiv:1710.04719 [math.DG]. 2017.

[GG18] P. Gaspar and M. Guaraco. "The Allen-Cahn equation on closed manifolds". Calculus of Variations and Partial Differential Equations 57(4) (2018), pp. 1-42.

[GT98] D. Gilbarg and N. Trudinger. Elliptic Partial Differential Equations of Second Order. Grundlehren der mathematischen Wissenschaften. Springer, 1998.

[Gua18] M. Guaraco. "Min-max for phase transitions and the existence of embedded minimal hypersurfaces". Journal of Differential Geometry 108(1) (2018), pp. 91-133.

[HT00] J. Hutchinson and Y. Tonegawa. "Convergence of phase interfaces in the van der Waals-Cahn-Hilliard theory". Calculus of Variations and Partial Differential Equations 10(1) (2000), pp. 49-84.

[Hut86] J. Hutchinson. "Second fundamental form for varifolds and the existence of surfaces minimising curvature". Indiana University Mathematics Journal 35(1) (1986), pp. 45-71.

[Ilm93] T. Ilmanen. "Convergence of the Allen-Cahn equation to Brakke's motion by mean curvature". Journal of Differential Geometry 38(2) (1993), pp. 417-461. 
[Le11] N. Le. "On the second inner variation of the Allen-Cahn functional and its applications". Indiana University Mathematics Journal 60 (2011), pp. 1843-1856.

[Le15] N. Le. "On the second inner variation of Allen-Cahn type energies and applications to local minimizers". Journal des Mathématiques Pures et Appliquées 103 (2015), pp. 1317-1345.

[LS18] N. Le and P. Sternberg. Asymptotic behavior of Allen-Cahn type energies and Neumann eigenvalues via inner variations. arXiv:1805.02604 [math.AP]. 2018.

[MN16] F. C. Marques and A. Neves. "Morse index and multiplicity of min-max minimal hypersurfaces". Cambridge Journal of Mathematics 4(4) (2016), pp. 463-511.

[Pit81] J. Pitts. Existence and Regularity of Minimal Surfaces on Riemannian Manifolds. Vol. 27. Mathematical Notes. Princeton University Press, 1981.

[Sim84] L. Simon. Lectures on Geometric Measure Theory. Proceedings of the Center for Mathematical Analysis. Australian National University, 1984.

[SS81] R. Schoen and L. Simon. "Regularity of stable minimal hypersurfaces". Communications on Pure and Applied Mathematics 34(6) (1981), pp. 741-797.

[SSY75] R. Schoen, L. Simon, and S.-T. Yau. "Curvature estimates for minimal hypersurfaces". Acta Mathematica 134(1) (1975), pp. 275-288.

[Ton05] Y. Tonegawa. "On stable critical points for a singular perturbation problem". Communications in Analysis and Geometry 13(2) (2005), pp. 439-459.

[TW12] Y. Tonegawa and N. Wickramasekera. "Stable phase interfaces in the van der Waals-Cahn-Hilliard theory". Journal für die reine und angewandte Mathematik (Crelles Journal) 2012(668) (2012), pp. 191-210.

[Wic14] N. Wickramasekera. "A general regularity theory for stable codimension 1 integral varifolds". Annals of Mathematics 179(3) (2014), pp. 843-1007.

[WW18] K. Wang and J. Wei. Finite Morse index implies finite ends. arXiv:1705.0683 [math.AP] To appear in Communications on Pure and Applied Mathematics. 2018.

Centre for Mathematical Sciences, University of Cambridge, Cambridge CB3 0WB, United Kingdom

E-mail address: f.l.hiesmayr@maths.cam.ac.uk 